\title{
Finite element modeling of powder bed fusion at part scale by a super-layer deposition method based on level set and mesh adaptation
}

\author{
Yancheng Zhang1 \\ Mines ParisTech, PSL Research University, Centre de Mise en Forme des Matériaux (CEMEF), \\ UMR CNRS 7635, Sophia Antipolis, France \\ e-mail: yancheng.zhang@mines-paristech.fr
}

\section{Charles-André Gandin}

Mines ParisTech, PSL Research University, Centre de Mise en Forme des Matériaux (CEMEF), UMR CNRS 7635, Sophia Antipolis, France

e-mail: charles-andre.gandin@mines-paristech.fr

\section{Michel Bellet}

Mines ParisTech, PSL Research University, Centre de Mise en Forme des Matériaux (CEMEF), UMR CNRS 7635, Sophia Antipolis, France

e-mail: michel.bellet@mines-paristech.fr

\begin{abstract}
A super-layer deposition method is developed for $3 D$ macroscopic finite element modeling of heat transfer at part scale during the powder bed fusion $(P B F)$ process. The proposed super-layer strategy consists of the deposition of batches of several layers. The main consideration is to deal with the effective heating times and with the inter-layer dwell time in a reasonable way. The material is deposited at once for each super-layer thanks to level-set and mesh adaptation methods, while the energy input is prescribed, either by respecting the layer-by-layer thermal cycle, or in a single thermal load. The level set method is used twice: first to track the interface between gas and the successive super-layers of powder bed and; second to track the interface between the part in construction and the non-exposed powder. To preserve simulation accuracy, adaptive remeshing is used to maintain a fine mesh near the evolving construction front during the process. Simulation results obtained by means of this super-layer method are presented and discussed by comparison with those obtained by layer-by-layer strategy, considered here as a reference. It is shown that, when respecting certain conditions, temperature evolutions and distributions approaching the reference ones can be obtained with significant savings on computation time. Assessment is first performed on simple part, then on a more complex configuration.
\end{abstract}

Keywords: additive manufacturing, powder bed fusion, finite element modeling, super-layer deposition, level-set, mesh adaptation

\footnotetext{
${ }^{1}$ Corresponding author.
} 


\section{INTRODUCTION}

2 Due to its efficient and flexible capabilities, additive manufacturing (AM) is at the heat of a

3 revolution in manufacturing processing. Powder bed fusion (PBF) is one of the numerous AM 4 processes, in which a moving heat source is used to selectively melt and solidify powder material to form a three-dimensional (3D) object, layer after layer. PBF consists of either electron beam powder bed fusion (EB-PBF), or laser powder bed fusion (L-PBF) [1]. These processes allow printing complex multifunctional parts in a single manufacturing step, which was not possible through traditional technologies. Moreover, different materials such as metals, ceramics and polymers are currently being evaluated, including combinations of different materials [2]. As a result, PBF has attracted both research and industry attention. To avoid the standard trial-and-error experimental approach, numerical models have been developed during the recent years, to simulate thermal and mechanical responses during and after the construction process, with the objective of reducing the time-to-market and saving research and development expenses.

Up to now, numerical models at different scales have been proposed to simulate L-PBF. At the scale of powder particles, Körner et al. [3] proposed a 2D lattice Boltzmann model to study melting and solidification of a randomly packed powder bed under the irradiation of a Gaussian beam. This model allows studying the influence of powder density, the stochastic effect of a randomly packed powder bed, capillary, wetting and balling phenomena. Khairallah et al. [4] presented a high-fidelity model for L-PBF by considering surface tension, Marangoni effect, recoil pressure in the melt pool. This model is able to capture the effect of strong dynamic melt flow on the generation of porosity, material spattering, and denudation. To further study such defects, Bayat et al. [5] also developed a high-fidelity numerical model based on the finite volume method (FVM) at track scale, in which the keyhole-induced porosities along with their initiating mechanisms were analyzed during single track L-PBF of a Ti-6Al-4V alloy. Moreover, the model was extended to multi-track/multi-layer by Bayat et al. [6] for predicting the formation and evolution of the porosities. By the investigated process parameters, the results show that porosities mainly appear in between the tracks, due to lack of fusion of the particles. To increase the computation efficiency for predicting melting/solidification process during L30 PBF, the powder bed may also be considered as a continuum, instead of using an explicit 31 description of the numerous particles [7,8]. This supposes the definition of equivalent properties for the powder-bed equivalent medium, e.g. equivalent density and thermal conductivity for 
1 heat transfer. A compressible formulation of the Navier-Stokes equations is then used to

2 account for the severe density increase during the transformation of the powder continuum into

3 a dense liquid phase, and further simulate fluid flow in the melt pool [7,8]. To ensure the stability and accuracy of the hydrodynamic analysis, the time step must be controlled and maintained to small values. This model also encompasses a non-linear thermal resolution developed by Saad et al. [9] to predict the temperature field evolution while accounting for fusion and solidification latent heat effects. Despite their pertinence at the scale of one or several deposited tracks, all previously mentioned approaches cannot address simulations at part scale, as this would generate prohibitive computation times [10].

With the goal to simulate the construction of entire parts, macroscopic approaches are the most appropriate and 3D finite element methods (FEM) are generally adopted. However, model predictions are affected by both inaccuracy and uncertainty [11]. Model inaccuracy arises from modelling assumptions at different stages, e.g. simplification of the thermal heat source, ignorance of powder material. Model uncertainty is a resultant of various sources of uncertain knowledge of material properties and process parameters. Zhang et al. [12] proposed a modeling strategy consisting in decomposing each deposited layer in a series of fractions considering the laser scanning path. When proceeding in this way, the local temperature evolution can be correctly predicted in a reasonable computation time. Moreover, the nonexposed powder is also considered around the built part during the construction process, providing more realistic conditions for the prediction of temperature distribution. Besides FEM, an efficient numerical method is also proposed by Zhang et al. [13] to simulate at part scale by considering laser scan path, which allows addressing parts with arbitrary complexity.

To further increase the computational efficiency and address the simulation of large parts by FEM, the layer-by-layer strategy has been adopted. An example can be found in the work of Chiumenti et al. [14], in which the temperature plot has an average evolution of the temperature field of the layer, but the CPU-time is notably reduced compared with the reference simulation by hatch-by-hatch strategy. It should be mentioned that the layer-by-layer method is a simplified model compared to the so-called "high-fidelity" analysis in which the exact scanning strategy is reproduced. Instead of this, the layer-by-layer approach (or approaches by fraction of layer) directly addresses each deposited layer as a whole (respectively portions of a whole 31 layer). It has been noted that [15], apart in the close neighborhood of the construction front, layer-based approaches can reliably model the transient physical effects at part scale, during the manufacturing process, e.g. comparable temperature gradients, and the temperature cycling of 
1 freshly deposited material, which gives access to the prediction of solid-state metallurgical 2 phase transformations, including their consequences in terms of distortions and residual stress.

3 However, such layer-by-layer calculations can still be too costly for parts made of thousands of 4 layers. This is why so-called super-layer or meta-layer strategies have emerged in the literature $[14,16,17,18,19,20,21]$. A super-layer consists of a certain number of layers, the deposition of which is modelled in a single operation. In the work of Chiumenti et al. [14], a homogeneous energy density is adopted, in which the elongated exposure time for the heat source is considered as one-time step for the thermal analysis. Similar work is done by Bayat et al. [20], while a short exposure time is proposed for the heat source of the super-layer method. Superlayers may be quite big, for instance, $\mathrm{Li}$ et al. [21] proposed a numerical model to predict residual stress and distortion while depositing 50 layers at a time (a $1.5 \mathrm{~mm}$ thick super-layer). Doing so, simulation times are significantly reduced and thermomechanical analyses can be more easily assessed. In above references, the simulation strategy for energy deposition is usually not clearly discussed. Concerning the assessment of heat transfer calculation, only final temperature distributions are generally given, while temperature evolutions of at selected locations are either ignored or not compared with those of corresponding results obtained by either layer-by-layer methods, or high-fidelity methods. Moreover, the limits of validity for such super-layer methods are not properly identified. In view of performing predictive simulations including thermo-metallurgical and thermo-mechanical computations, more indepth investigations are required, which is the objective of the present contribution.

In this paper, the super-layer method is discussed and evaluated in the restricted context of thermal analysis and in the framework of the level-set method and mesh adaptation: $i$ - the material is deposited by successive super-layers, the energy input being applied for each superlayer by a uniform heat source while respecting a temporal cycle adapted to the number of layers contained in each super-layer, $i i$ - mesh adaptation is applied, preserving a fine mesh at upper and lower boundaries of the super-layer, while generating a coarse mesh far from the heating zone, and iii- the non-exposed powder and its role on heat flow at part scale is considered during the deposition process. For a simple manufacturing configuration, temperature evolution and distribution are presented and discussed by comparison with reference results defined as the layer-by-layer strategy. Then, an application example for a more complex manufacturing configuration is given in the last section. 


\section{LEVEL SET AND DYNAMIC ADAPTIVE MESH CONTROL}

2 The numerical simulation is operated on a single three-dimensional domain $\Omega$, which is

3 discretized with tetrahedral finite elements in a non-structured manner. At any instant $t$ of the construction process, $\Omega$ encompasses a material domain and a gas domain. The material domain consists itself of two domains: the dense material (the substrate and the already constructed workpiece), and the non-consolidated powder, as schematized in Fig. 1. In the present model simulation, the interfaces separating the different domains are described using the level set formulation [10]. As shown in Fig.1, a first level set function $\psi$ (level set 1 in the figure) tracks the interface between the material domain and the gas domain. This function is the signed distance to this interface, and takes the value 0 at the interface (contour in red color in the figure).

11 It is positive in the gas domain and negative in the material domain. Material deposition is realized by updating function $\psi$ with time as follows:

$$
\psi^{t+\Delta t}=\psi^{t}-\Delta z
$$

14 Assuming a constant layer thickness $\Delta z^{l}$ during the construction process, the level set 15 increment $\Delta z$ can be written as $\Delta z=n^{l} \Delta z^{l}$. The layer-by-layer modelling approach corresponds to $n^{l}=1$, while we have $n^{l}>1$ for a super-layer approach (deposition of $n^{l}$ 17 layers in a single batch).

18 Another level set function, $\varphi_{\text {geo. }}$, is used to track the geometry of the part to be built: $\varphi_{\text {geo. }}>$ 0 in the part and $\varphi_{\text {geo. }}<0$ outside (Fig. 1, all line contours in blue color). Concerning the 20 already constructed part, it is defined by a third level set function, $\varphi$ (level set 2 in the figure) 21 for which $\varphi=0$ (Fig. 1, blue lines contours) represents the interface between the already constructed part, and the non-exposed powder or the gas. Function $\varphi$ is defined by combining the level set function of the part geometry, $\varphi_{\text {geo. }}$, and the pre-defined level set $\psi$ : 


$$
\varphi=\min \left(\varphi_{g e o,}, \psi\right)
$$

1 As a result, $\varphi$ is positive in the workpiece domain (already constructed workpiece and substrate)

2 and negative in powder and gas domains.

3 Regarding accuracy and efficiency of simulation, the local mesh size and the total number of

4 elements need to be controlled, based on the two level sets $\psi$ and $\varphi$. In the present work,

5 inhomogeneous mesh is adopted to satisfy the requirements of dynamic adaptive mesh refining

6 and coarsening. The mesh is refined around the interface defined by $\varphi=0$ within the deposited

7 layer, colored in yellow in Fig. 1. On the contrary, the mesh is progressively coarsened when

8 increasing the distance from the interface $\psi=0$. During the construction process, the interface

$9 \quad \psi=0$ is displaced upwards in the vertical direction using Eq. 1. The mesh needs to be updated

10 accordingly, i.e. at the same frequency as the interface with the gas domain evolves. The main

11 idea is to define two mesh metrics. Firstly, a background mesh metric on the whole domain $\Omega$

12 is defined by the level sets $\psi$ and $\varphi$, which generates a homogeneous coarse mesh far from the

13 two interfaces and inhomogeneous fine mesh around them. Secondly, a local mesh metric in

14 the vicinity of the construction front is defined by $\varphi_{\text {Fine }}=\min \left(\varphi, \psi+\Delta z^{s l},-\psi\right)$, which

15 generates a fine and inhomogeneous mesh in this area. The final metric is obtained by the

16 intersection of the above two metrics. The specific mesh control algorithm is explained in a

17 previous paper by Zhang et al. [12]. 


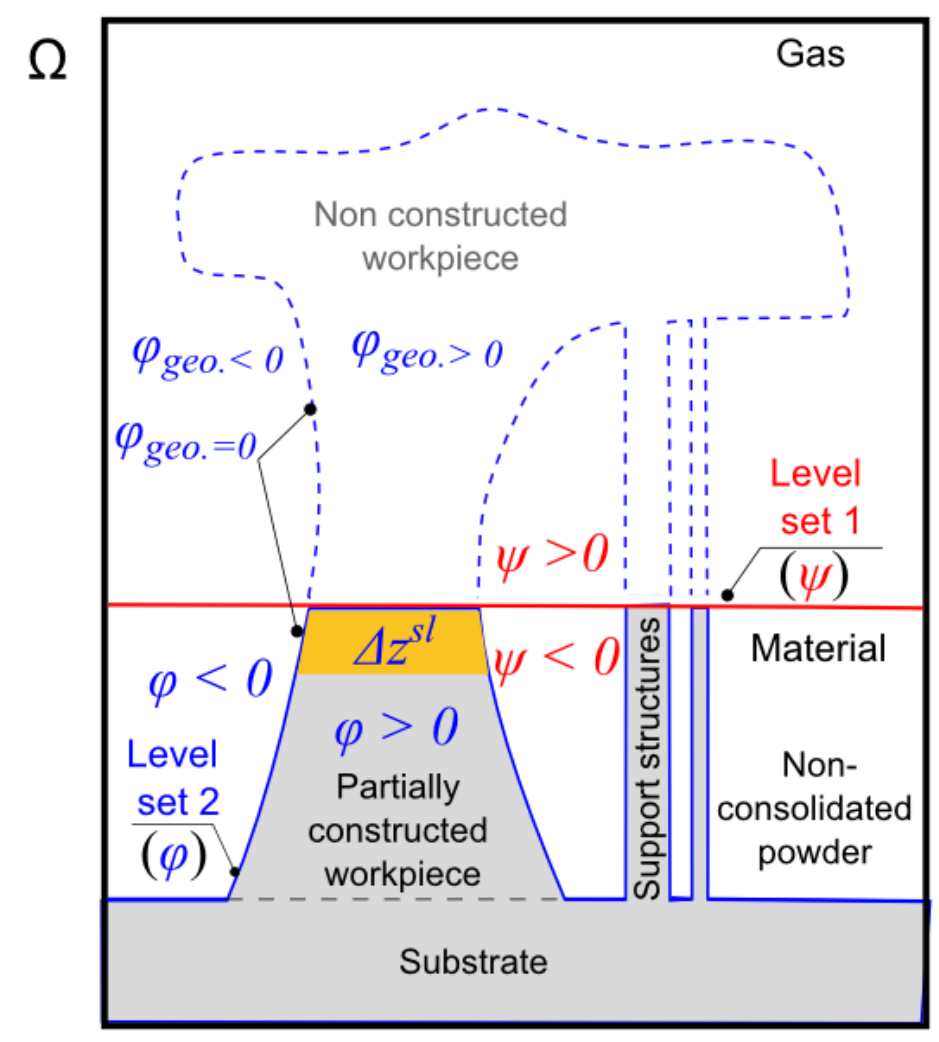

\section{POWDER AND ENERGY INPUT IN THE SUPER-LAYER APPROACH}

4 As already introduced in Section 2, the material can be deposited by a series of super-layers, 5 each of them containing a certain number of layers, $n^{l}>1$, and being deposited instantaneously. The main objective of the super-layer strategy is to reach significant savings in computation cost as will be studied hereafter when solving a heat transfer problem. The basic reason is that each addition using Eq. (1) requires performing a remeshing procedure. Therefore, decreasing the number of depositions by using the super-layer strategy naturally decreases the number of remeshing operations, hence saving computation time. Nonetheless, energy inputs to the system with a super-layer method have to be rigorously controlled so as to be representative of the layer-by-layer strategy, the latter being used as the reference case in the following.

14 The layer-by-layer method is first reviewed. Two super-layer methods are then introduced. Both super-layer methods consider deposition of several layers instantaneously with total thickness $\Delta z^{s l}$, corresponding to the summation of $n^{l}(>1)$ layers. The super-layer methods differ in the number of heating steps, $n^{h}$, which are performed during each super-layer deposition. The first method is named "multi-shot". Energy deposition is imposed in the whole volume of the super- 
1 layer in a number of heating steps $n^{h}=n^{l}$. The second super-layer method is named "one-shot"

2 as all heating steps involved in the successive deposition of $n^{l}$ layers are merged in a single 3 equivalent heating step, so $n^{h}=1$. Considering $\Delta t^{l}$ as the time required for a single layer 4 addition in the process (recoating time, plus laser scanning time), Fig. 2 schematically illustrates 5 the differences between the different strategies - layer-by-layer method, "multi-shot" and "one6 shot" multi-layer methods - on a time sequence of $5 \Delta t^{l}$, during which five layers are deposited.
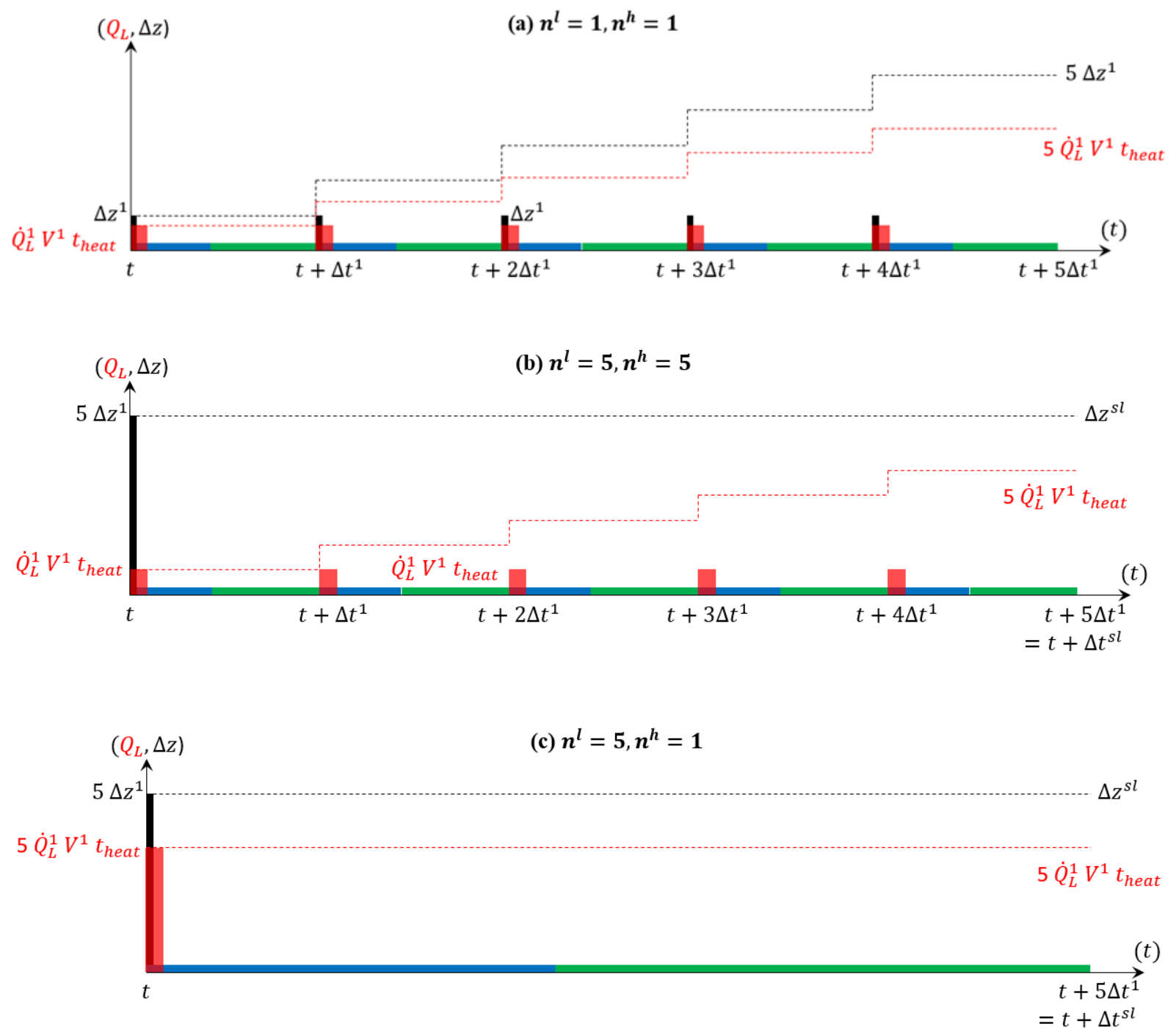

Fig. 2. Schematic time sequence to model a series of 5 layer additions, with indication of instants for material and energy supply for 3 methods: (a) layer-by-layer, with $n^{l}=1$ and $n^{h}=1$; (b) "multi-shot" super-layer, with $n^{l}=5$ and $n^{h}=5$; and (c) "one-shot" super-layer, with $n^{l}=5$ and $n^{h}=1$ ( $n^{l}$ is the number of layers within each super-layer, $n^{h}$ is the number of heating steps for each super-layer) 


\section{$1 \quad 3.1 \quad$ The layer-by-layer method}

2 The layer-by-layer method consists in adding single layers of thickness $\Delta z^{l}$ while solving the

3 heat flow problem repeatedly and incrementally during the time interval $\Delta t^{l}$. The heating time

$4 t_{\text {heat }}$ is then evaluated as the effective time during which the material is locally exposed to the

5 laser beam, so that it can be reasonably expressed by:

$$
t_{\text {heat }}=\frac{\phi_{L}}{v_{L}}
$$

6 where $\phi_{L}$ is the diameter of the laser beam and $v_{L}$ its travelling velocity, both being assumed

7 constant over all layers. Knowing the area of the $l^{\text {th }}$ layer (effective laser-scanned area within

8 a considered powder layer), $S^{l}$, and the hatch spacing between adjacent tracks, $H_{S}$, an

9 estimation of $t_{\text {scan }}^{l}$ is given by:

$$
t_{\text {scan }}^{l}=\frac{S^{l}}{H_{S} v_{L}}
$$

10 Consequently, the following energy balance can be derived for each layer:

$$
P_{L}(1-R) t_{\text {scan }}^{l}=\dot{Q}_{L}^{l} V^{l} t_{\text {heat }}
$$

11 where $P_{L}$ is the laser power and $R$ is the reflection coefficient of the laser radiation at the surface 12 of the material. The left-hand side of Eq. (5) represents the energy delivered by the heat source 13 during the scan time $t_{\text {scan }}^{l}$ of each layer. The right-hand term expresses the energy absorbed by 14 the material within the scanned volume $V^{l}=\Delta z^{l} S^{l}$, during the heating time, $t_{\text {heat }}$. So injecting 15 Eqs (3) and (4) in Eq. (5), the expression of the volume heat source $\dot{Q}_{L}^{l}$ to be delivered in a 16 single layer during its heating time can be obtained:

$$
\dot{Q}_{L}^{l}=\frac{P_{L}(1-R)}{H_{S} \phi_{L} \Delta z^{l}}
$$

17 It is worth considering the ratio $t_{\text {heat }} / t_{\text {scan }}^{l}$ given by:

$$
t_{\text {heat }} / t_{\text {scan }}^{l}=H_{S} \phi_{L} / S^{l}
$$

18 Assuming $\phi_{L}$ and $H_{S}$ of the order of $100 \mu \mathrm{m}$ and $S^{l}$ of the order of hundreds of $\mathrm{mm}^{2}$, then the 19 ratio is of the order of $10^{-4}$. This short heating step is assumed to take place at the beginning 
1 of each layer deposition during the modelling time sequence, as shown in Fig. 2(a): red bars.

2 The cooling time $t_{c o o l}^{l}$ is then given by:

$$
t_{\text {cool }}^{l}=t_{\text {scan }}^{l}+t_{d w e l l}^{l}-t_{\text {heat }}
$$

3 where $t_{d w e l l}^{l}$ is the time required to spread a new powder layer.

4 In the schematics proposed in Fig. 2(a), constant thickness and section area are used over 5 5 cycles so a total deposited thickness of $5 \Delta z^{l}$ is reached. This takes place over $5 \Delta t^{l}$ where $\Delta t^{l}$ characterizes the time period for a single layer deposition. It is the summation of the scan time

of the heat source, $t_{\text {scan }}^{l}$, plus the corresponding dwell time, $t_{d w e l l}^{l}: \Delta t^{l}=t_{\text {scan }}^{l}+t_{d w e l l}^{l}$. By 8 Eq.(8), $\Delta t^{l}$ can be expressed as $\Delta t^{l}=t_{\text {heat }}+t_{\text {cool }}^{l}$, from the numerical point of view. With the 9 volume heat source $\dot{Q}_{L}^{l}$ for each deposited layer on a volume $V^{l}$, the total energy input is $5 \dot{Q}_{L}^{l} V^{l} t_{\text {heat }}$ after 5 cycles.

\section{2 "Multi-shot" super-layer method}

The main difference with the layer-by-layer method obviously lies in the material deposition, as $n^{l}$ layers are instantly deposited in a single batch. For instance, in Fig. 2(b), this incremental batch of material is directly imposed with a thickness $\Delta z^{s l}=5 \Delta z^{l}$ and the construction level (Eq. (1)) is maintained constant up to the next super-layer deposition. However, in the "multishot" method, the thermal load through the time cycle for the super-layer is in accordance with the effective time cycling of the real process. Consequently, there are as many heating computation steps as the number of layers in the super-layer $\left(n^{h}=n^{l}\right)$, and each heating step is followed by its corresponding cooling time. Such a temporal sequence is illustrated by Fig. 2(b). Heat flow is treated in a very similar way as in Fig. 2 (a), with a total energy input progressively added over time to reach the same amount after 5 cycles, $5 \dot{Q}_{L}^{l} V^{l} t_{\text {heat }}$. Accordingly, Eq. (5) can be rewritten

$$
P_{L}(1-R) t_{s c a n}^{s l}=\dot{Q}_{L}^{s l} V^{s l} t_{\text {heat }}^{s l}
$$

As previously, assuming constant thickness and section area for each layer, the total scan time rewrites $t_{\text {scan }}^{\text {sl }}=\sum_{l=1}^{n^{l}} t_{\text {scan }}^{l}=n^{l} t_{\text {scan }}^{l}$ and the total heating time rewrites $t_{\text {heat }}^{\text {sl }}=\sum_{l=1}^{n^{h}} t_{\text {heat }}=$ $n^{h} t_{\text {heat }}$ and can still be evaluated using Eqs. (4) and (3), respectively. The total volume of the super-layer writes $V^{s l}=\sum_{l=1}^{n^{l}} S^{l} \Delta z^{l}=\Delta z^{l} \sum_{l=1}^{n^{l}} S^{l}$. As a consequence, the new expression of the volume heat source $\dot{Q}_{L}^{s l}$ (considered as identical at each heating) becomes: 


$$
\dot{Q}_{L}^{s l}=\frac{P_{L}(1-R)}{H_{S} \phi_{L} \Delta z^{l} n^{h}}
$$

1 The difference with respect to Eq. (6) is the presence of $n^{h}$ in the denominator that expresses a

2 lower power density, in coherence with the multiple heating steps of the "multi-shot" super-

3 layer method. The expression of the cooling time can then be deduced:

$$
t_{\text {cool }}^{s l}=\Delta t^{s l}-t_{\text {heat }}^{\text {sl }}=\sum_{l=1}^{n^{l}}\left(t_{\text {scan }}^{l}+t_{\text {dwell }}^{l}\right)-n^{h} t_{\text {heat }}
$$

4 Note that in Fig. 2(b) a constant cooling time over each energy input is also assumed for the

5 schematics by $t_{c o o l}^{s l} / n^{l}=\left(t_{d w e l l}^{l}+t_{\text {scan }}^{l}-t_{\text {heat }}\right)$, which is the same as Eq. (8).

$6 \quad 3.3$ "One-shot" super-layer method

7 The super-layer method with a one-shot heating is illustrated in Fig. 2(c). Not only the material 8 input $\Delta z^{s l}=5 \Delta z^{l}$ is achieved at the beginning of $\Delta t^{s l}$, but the same is also done for the energy 9 input. Such a one-shot energy input $\left(n^{h}=1\right)$ has to be consistent with the global laser energy 10 transmitted to the workpiece under construction. For that, Eq. (9) can be rewritten

$$
P_{L}(1-R) t_{s c a n}^{s l}=\dot{Q}_{L}^{s l} V^{s l} t_{\text {heat }}
$$

11 where $t_{s c a n}^{s l}$ and $V^{s l}$ take the same summation form as in Eq. (9), $t_{\text {heat }}$ being still expressed by 12 Eq. (3) due to the one-shot method. The expression of the volume heat source $\dot{Q}_{L}^{s l}$ is then the 13 same as for the layer-by-layer method (Eq. (6)):

$$
\dot{Q}_{L}^{s l}=\frac{P_{L}(1-R)}{H_{S} \phi_{L} \Delta z^{l}}
$$

14 The cooling time is now calculated as follows:

$$
t_{\text {cool }}^{\text {sl }}=\Delta t^{s l}-t_{\text {heat }}=\sum_{l=1}^{n^{l}}\left(t_{\text {scan }}^{l}+t_{d w e l l}^{l}\right)-t_{\text {heat }}
$$

15 In the light of the comparison between Eqs. (10)-(11) and (13)-(14), the main weak point of the 16 "one-shot" method can be anticipated. The system will cool down during quite a longer-time 17 interval between two successive heating steps $\Delta t^{s l}$ compared to the "multi-shot" method where cooling is multiple $\left(n^{h}>1\right)$. The risk for larger amplitude between successive max and min 
1 temperatures of a characteristic thermal cycle will thus be exacerbated compared to the 2 multi-shot method.

3 The main objective of this paper is to compare the three methods and to identify the impact of 4 the number of layers per super-layer, $n^{l}$, as well as the number of heating steps, $n^{h}$, on the simulation results. As a result, better anticipation is expected on possible compromises between computation time and prediction accuracy. The methods are compared regarding temperature evolutions and distributions for simple and complex cases in Sections 5 and 6. Before performing the numerical simulations, some comments on the resolution of the heat transfer equation in the super-layer context are given in Section 4.

\section{HEAT TRANSFER EQUATION}

11 The standard heat transfer equation is considered for the thermal analysis:

$$
\rho c_{p} \frac{d T}{d t}-\nabla \cdot(k \nabla T)=\dot{Q}_{L}
$$

12 with $T$ the temperature, $\rho$ the density, $c_{\mathrm{p}}$ the specific heat, $k$ the thermal conductivity and $\dot{Q}_{L}$ the volumetric heat source defined in Section 3, either using $\dot{Q}_{L}^{l}$ given by Eq. (6), or using $\dot{Q}_{L}^{s l}$ 14 given by Eqs. (10) and (13). The regions where melting and solidification occur are very close to each other typically three to four times the beam diameter $\phi_{L}$, which is a very small distance with respect to the part size. This justifies that the latent heat of fusion and solidification can be ignored when solving heat transfer at part scale, which is also proved by the work of Shen and Chou [22]. Consequently, during the numerical simulation the weak form makes linear the finite element resolution of the set of equations deduced from Eq. (15), and this allows performing a single linear resolution of this system of equations at each time step. Besides, it should be mentioned that the conductivity of the powder domain (powder non-exposed to the laser interaction) is calculated by the relationships proposed by Sih and Barlow [23, 24], in which the porosity of the powder bed is considered.

The first simulation domain is a 3D parallelepiped, in which a cylinder is printed (Fig. 3). As seen in this perspective view, the simulation domain, $80 \mathrm{~mm}$ high, includes gas at the top, a 10 mm-thick substrate at the bottom, and the cylinder part to be built in between, of height $\Delta z_{p, \max }$. Along the bottom face of the domain, a convective heat exchange is considered, with an air 
1 temperature $T_{\text {air }}$ and a heat exchange coefficient $h_{\text {conv }}$. The unit outward normal vector being

2 denoted $\boldsymbol{n}$, the extracted heat flux is expressed by:

$$
-k \nabla T \cdot \boldsymbol{n}=h_{\text {conv }}\left(T-T_{\text {air }}\right)
$$

3 A similar equation, with the conductive coefficient $h_{\text {cond }}$ instead of $h_{\text {conv }}$, and $T_{\text {ext }}$ instead of $T_{\text {air }}$,

4 is considered for heat extraction between the lateral surfaces of the domain and the machine environment, while an adiabatic boundary condition is considered at the top surface of the domain. It should be mentioned that only thermal diffusion is considered along the evolving interface between the top of the constructed part and the gas domain. The initial temperature in the system is set as $T_{0}$.

9 In the present context, the temperature solution issued from Eq. (15) may be affected by 10 temporal or/and spatial oscillations. The reasons for this lie in the diffusive thermal shock arising from high and localized energy inputs close to a material in the powder state, showing fairly low heat conductivity. In order to control such undesirable effects, different methods can be used, among which the asynchronous resolution of Eq. (15), or the cond-split scheme as proposed by Fachinotti \& Bellet [25]. Another strategy consists in artificially extending the heating stage, by multiplying the right-hand side of Eq. (3) by a constant factor $\alpha$ greater than 1.

$$
t_{\text {heat }}=\alpha \frac{\phi_{L}}{v_{L}}
$$

17 The latter methodology offers two advantages. First, considering Eqs. (5) and (6), this clearly 18 induces a decrease in the volume heat source $\dot{Q}_{L}: \alpha$ will appear in the denominator of the 19 expressions defining $\dot{Q}_{L}$. Second, such an increase of $t_{\text {heat }}$ allows increasing the computation time step $\Delta t$. This is also favorable as diffusive thermal shock occurs when the diffusion length during a time increment is lower than the mesh size $h_{m s h}$ :

$$
\sqrt{\frac{k}{\rho c_{p}} \Delta t}<h_{m s h}
$$

As already studied in reference [12], the value of $\alpha$ may be chosen relatively large, typically up 23 to several hundred or thousand, while still maintaining a very small ratio $t_{\text {heat }} / t_{\text {scan }}^{l}$ without 24 impacting significantly the solution temperature field ( $\alpha$ can be determined by the maximum 
1 temperature reaching the liquidus at the heated super-layer, for example). This is why the 2 heating times in Fig. 2 are only schematics.

\section{MODEL VERIFICATION}

4 First, to test the proposed super-layer model with respect to energy conservation, a simple test 5 consisting of the construction of a square pillar built on a substrate [12] was first adopted with 6 adiabatic bottom and lateral boundaries. It was verified that the multilayer strategy has no 7 impact on the total energy input in the part under construction. It was found - but not related in 8 detail, nor documented here - that the difference in energy input between layer-by-layer and 9 super-layer methods was less than $0.53 \%$ for $n^{l}=5$, and less than $1.08 \%$ for $n^{l}=15$.

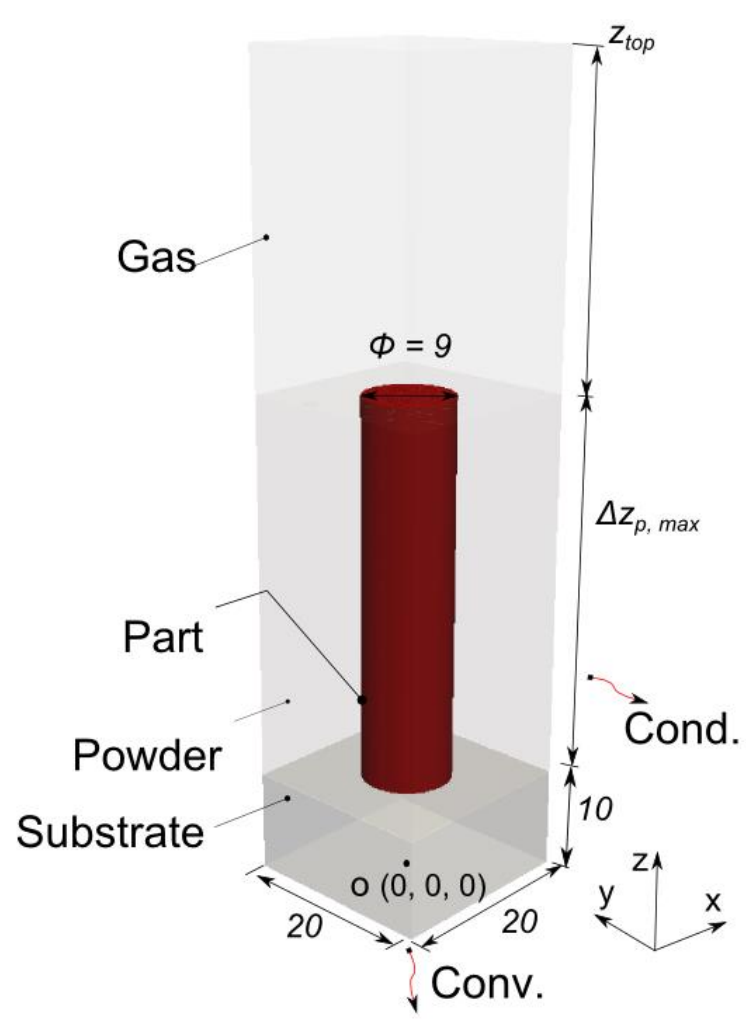

Fig. 3. Geometry of the simulation system (dimensions in $\mathrm{mm}$ )

12 Following this first check, our interest now focuses on the test described in Fig. 3. During the 13 construction, a $9 \mathrm{~mm}$-diameter cylinder is built at the top surface of a $10 \mathrm{~mm}$-thick substrate, 14 with an evolving height up to $\Delta z_{p, \max }$. This cylinder is surrounded by the non-exposed powder 15 (in light gray color) with a final top surface at $z_{p}=10+\Delta z_{p, \max }$. The gas region extends 
1 above, up to $z=z_{\text {top }}=80 \mathrm{~mm}$. Material properties, heat exchange and process parameters are

2 given in Table 1.

3 Table 1

4 Material properties, heat exchange and process parameters for the test case shown in Fig. 3 [7, 23, 24] Properties Workpiece Powder bed Argon gas

$(\mathrm{Ti}-6 \mathrm{Al}-4 \mathrm{~V}) \quad(\mathrm{Ar})$

\begin{tabular}{|c|c|c|c|c|}
\hline \multirow[t]{3}{*}{ Materials } & Density, $\rho\left[\mathrm{kg} \cdot \mathrm{m}^{-3}\right]$ & 4430 & 2480.8 & 1.3 \\
\hline & Thermal conductivity, $k\left[\mathrm{~W} \cdot \mathrm{m}^{-1} \cdot \mathrm{K}^{-1}\right]$ & 6.6 & 0.378 & 0.024 \\
\hline & Specific heat, $c_{p}\left[\mathrm{~J} \mathrm{~kg}^{-1} \cdot \mathrm{K}^{-1}\right]$ & 565 & 565 & 1000 \\
\hline \multirow{5}{*}{$\begin{array}{l}\text { Heat } \\
\text { exchange }\end{array}$} & Machine environment, $T_{\text {ext }}\left[{ }^{\circ} \mathrm{C}\right]$ & \multicolumn{3}{|c|}{25} \\
\hline & Initial temperature of $\Omega, T_{0}\left[{ }^{\circ} \mathrm{C}\right]$ & \multicolumn{3}{|c|}{25} \\
\hline & Ambient temperature, $T_{\text {air }}\left[{ }^{\circ} \mathrm{C}\right]$ & \multicolumn{3}{|c|}{25} \\
\hline & Conductive coefficient, $h_{\text {cond }}\left[\mathrm{W} \cdot \mathrm{m}^{-2} \cdot \mathrm{K}^{-1}\right]$ & \multicolumn{3}{|c|}{15} \\
\hline & Convection coefficient, $h_{\text {conv }}\left[\mathrm{W} \cdot \mathrm{m}^{-2} \cdot \mathrm{K}^{-1}\right]$ & \multicolumn{3}{|c|}{15} \\
\hline & Nominal power, $P_{L}[\mathrm{~W}]$ & \multicolumn{3}{|c|}{170} \\
\hline & Scan speed, $v_{L}\left[\mathrm{~mm} \cdot \mathrm{s}^{-1}\right]$ & \multicolumn{3}{|c|}{1500} \\
\hline J acer & Reflection coefficient, $R$ & \multicolumn{3}{|c|}{0.3} \\
\hline Lase? & Diameter of laser beam, $\phi_{L}[\mu \mathrm{m}]$ & \multicolumn{3}{|c|}{73.6} \\
\hline & hatch spacing, $H_{S}[\mu \mathrm{m}]$ & \multicolumn{3}{|c|}{73.6} \\
\hline & Thickness of powder layer, $\Delta z_{l}[\mu \mathrm{m}]$ & \multicolumn{3}{|c|}{40} \\
\hline & Total deposited height, $\Delta z_{p, \max }[\mathrm{mm}]$ & \multicolumn{3}{|c|}{38.4 (960 layers) } \\
\hline & Scaling factor, $\alpha$ & \multicolumn{3}{|c|}{1230} \\
\hline & Inter-layer dwell time, $t_{d w e l l}^{l}[\mathrm{~s}]$ & \multicolumn{3}{|c|}{7} \\
\hline
\end{tabular}

5

65.1 Comparison of the proposed super-layer strategies

7 This verification is focused on temperature evolution, which is very important for the 8 metallurgical and mechanical analyses. For that purpose, the response of a temperature sensor 9 located at the bottom center of the cylinder (sensor A, location indicated in Fig. 4) is tested for 
1 different solution strategies: layer-by-layer $\left(n^{l}=1, n^{h}=1\right)$, super-layer by "one-shot" ( $n^{l}=$ $\left.25, n^{h}=1\right)$ and super-layer by "multi-shot" $\left(n^{l}=5, n^{h}=5\right)$. As the simulation of the whole construction - which consists of almost a thousand of very thin layers - requires a significant computation time, only the first 30 layers are studied here for comparison. The layer-by-layer strategy ( $n^{l}=1, n^{h}=1$, results in black color in Fig. 4) is considered as the reference case. When using the super-layer strategy, with $n^{l}=5$, the "one-shot" method ( $n^{h}=1$, thin red line) shows extra-high peak values due to a severe time concentration of the heat input, while the minimum values (obtained after dwell time) are similar to those of the reference curve. For the "multi-shot" method ( $n^{h}=5$, thick red line), it can be observed that except for the very first layers, the peak temperature values are quite close to the reference calculation, and the minimum values are always identical to the reference ones. What is even more important is that the repeated thermal cycles are very well reproduced by the "multi-shot" super-layer strategy.

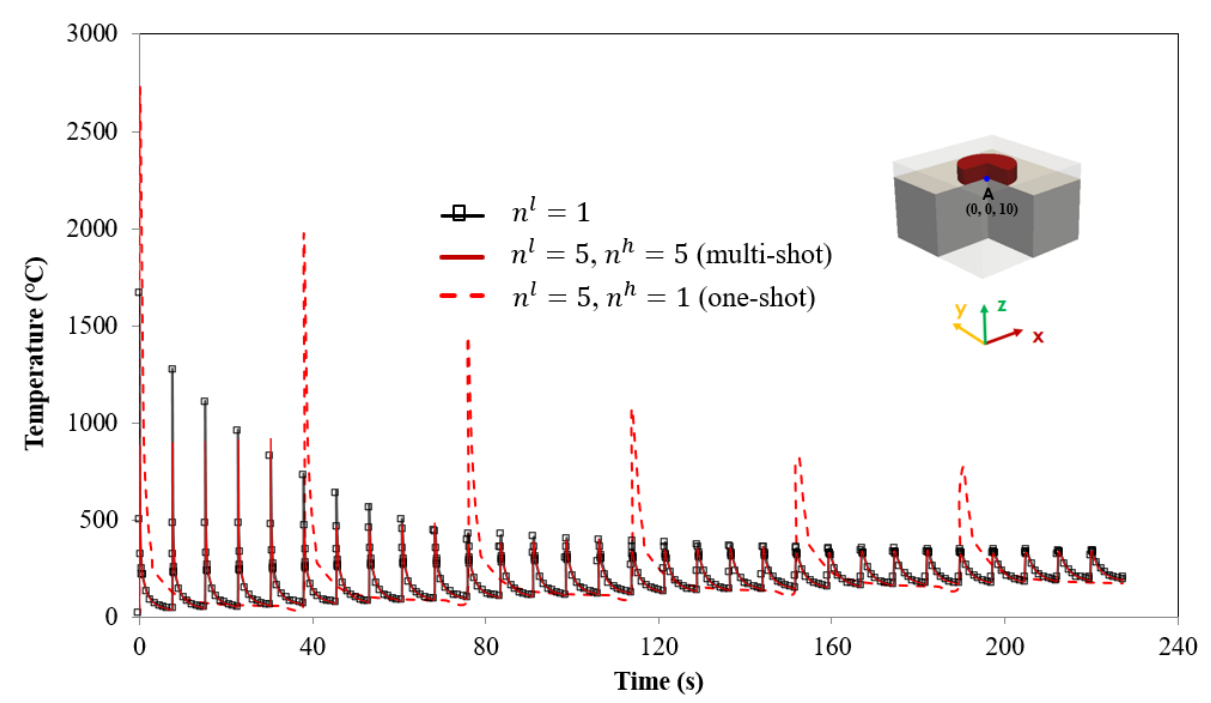

14 Fig. 4. Temperature evolution at location A during the deposition of the first 30 layers for the test case described by Fig. 3 and Table 1. Comparison is given between the layer-by-layer approach (thick black curve), the "one-shot" super-layer method (thin red line) and the "multishot" super-layer method (thick red line)

Beside temperature accuracy, the computation efficiency can be studied and is summarized in Table 2 for the 30-layer deposition simulated with 28 cores. To carry out the simulation using the layer-by-layer approach, considering layers of thickness $40 \mu \mathrm{m}$ for a domain size $20 \times 20 \times 80$ $\mathrm{mm}^{3}$, a non-structured initial mesh containing about 1.7 million tetrahedral elements is adopted for the adaptive mesh strategy. At the end of the 30-layer deposition, a non-structure mesh containing around 1.9 million tetrahedral elements is obtained. Compared to a reference computation time of 418 minutes $(\sim 7 \mathrm{~h})$, obtained with the layer-by-layer method, the multi- 
1 layer method, here with $\left(n^{l}=5\right)$, provides significant time reduction: 118 minutes $(\sim 2 \mathrm{~h})$ for 2 the "one-shot" method $\left(n^{h}=1\right)$, and 267 minutes $(\sim 4.5 \mathrm{~h})$ for the "multi-shot" method $\left(n^{h}=\right.$ $35)$.

\section{Table 2}

5 Computation time for the simulation of a 30-layer deposition with 28 core parallel computation

\begin{tabular}{|c|c|c|c|c|}
\hline & $n^{l}$ & 1 & 5 & 5 \\
\hline & $n^{h}$ & 1 & $\begin{array}{c}5 \\
\text { (multi-shot) }\end{array}$ & $\begin{array}{c}1 \\
\text { (one-shot) }\end{array}$ \\
\hline $\mathrm{Nu}$ & $\begin{array}{l}\text { ber of layers/ } \\
\text { uper-layers }\end{array}$ & 30 & 6 & 6 \\
\hline \multirow{2}{*}{$\begin{array}{c}\text { Final mesh } \\
\text { informatio } \\
n\end{array}$} & $\begin{array}{l}\text { Number of Nodes } \\
\left(\times 10^{6}\right)\end{array}$ & 0.33 & 0.33 & 0.33 \\
\hline & $\begin{array}{l}\text { Number of Elements } \\
\qquad\left(\times 10^{6}\right)\end{array}$ & 1.91 & 1.93 & 1.94 \\
\hline \multicolumn{2}{|c|}{ Computation time (min) } & 418 & 267 & 118 \\
\hline \multicolumn{2}{|r|}{ Time gain } & - & 1.56 & 3.54 \\
\hline
\end{tabular}

8 As the layer-by-layer deposition is too costly to simulate one thousand layers, the "multi-shot" 9 super-layer method with $n^{l}=5$ and $n^{h}=5$ will be considered as the reference case in the following sections, in which the robustness and precision of the proposed "multi-shot" and “one-shot" super-layer strategies are studied.

\subsection{Sensitivity analysis for $n^{l}$ and $n^{h}$}

13 Considering $n^{l}=5$ and $n^{h}=5$ as the reference case for the entire part construction, $n^{l}=5$ and $n^{l}=15$ are studied to verify the robustness and accuracy of the "multi-shot" and "one-shot" super-layer methods. Figure 5 shows the calculated temperature evolution at sensor A (defined in Fig. 4) during the construction process, and the temperature distribution (central vertical profile) after the dwell time following the last layer deposition. With the "multi-shot" method, the temperature evolutions calculated for the two different values of $n^{1}$ are found superimposed (Fig. 5 (a)) except in the very beginning of the construction process. On the contrary, big oscillations are found for the "one-shot" method. This confirms and extends the result obtained in the preliminary study. 
(a)
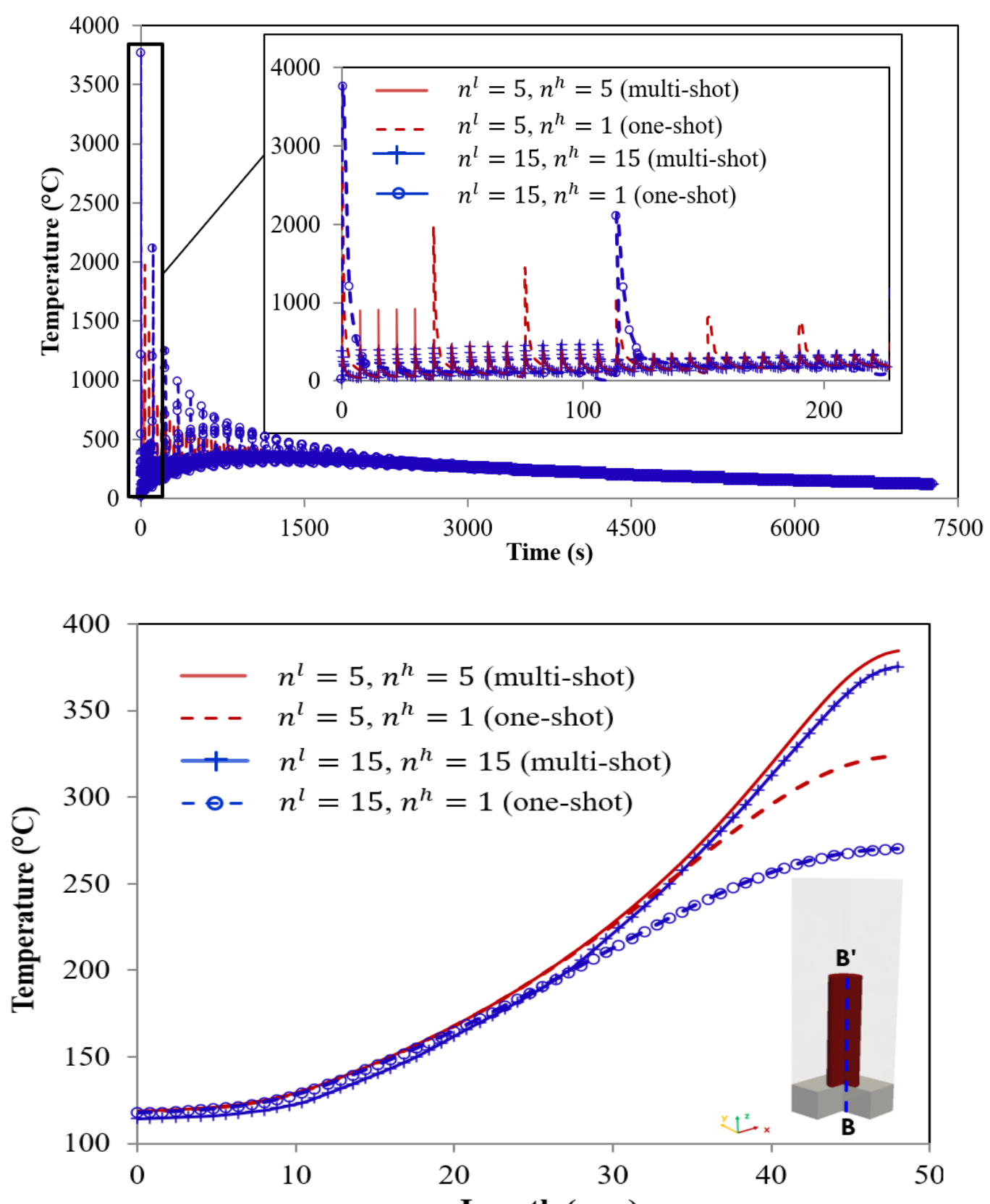

3 Fig. 5. Simulation of the construction of a vertical cylinder with the multi-shot super-layer

4 strategy, using two different values for $n^{1}$ with (a) temperature evolution at sensor $\mathrm{A}$ and (b)

5 temperature profile along the center line $\mathrm{BB}^{\prime}$ after the dwell time of the last layer

6 At the end of the process, after the final dwell time, the vertical temperature profile obtained

7 for $n^{l}=15$ and $n^{h}=15$ tends to overlap with that of the reference ( $n^{l}=5$ and $n^{h}=5$ ) (Fig.

85 (b)). In contrast, the "one-shot" method leads to discrepancies when compared to the "multi-

9 shot" method. Thus, the single heating strategy $\left(n^{h}=1\right)$, which imposes a strong energy power

10 within a short time according to Eq. (13), has a clear detrimental impact on the temperature

11 profile. The peaks are especially marked near the construction front and become lower far from 
1 the front. Large temperature differences are observed at point $B^{\prime}$ when using the "one-shot" 2 method $\left(n^{h}=1\right)$, reaching $60^{\circ} \mathrm{C}$ and $114^{\circ} \mathrm{C}$ (see Table 3) for $n^{l}=5$ and $n^{l}=15$, 3 respectively.

4 At the end of the construction, the calculated temperature distribution in the entire domain 5 including the non-exposed powder is shown in Fig. 6(a). By the mesh distribution in the yz 6 section plane, the fine mesh is kept along the constructed part to maintain the built geometry. 7 Moreover, the mesh distribution in the super-layer zone is given, defined by the red contour 8 line $\left(\varphi_{\text {Fine }}\right)$ in the zoomed inset, in which the fine mesh is generated around the interface of $\varphi_{\text {Fine }}$ and gradual coarse meshes inside and outside of the super-layer are given. By filtering the mesh in the gas and powder domains, Fig. 6(c) only shows the temperature distribution in

11 the constructed part. Actually, the temperature distribution is similar for the two cases $\left(n^{1}=5\right.$ and 15) of the "multi-shot" deposition, which is demonstrated by Fig. 5(b). (a)

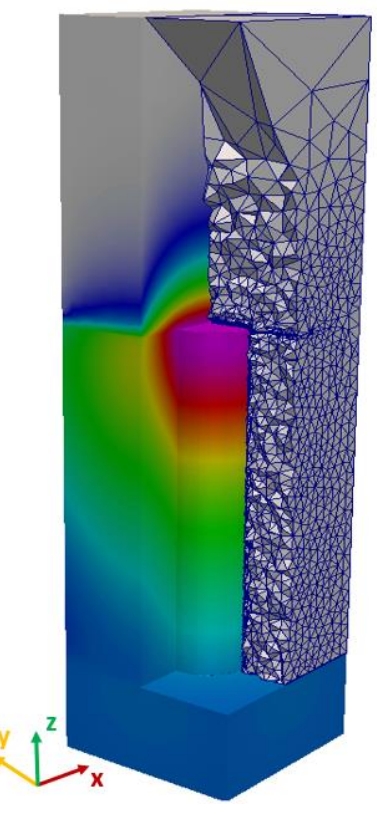

(b)

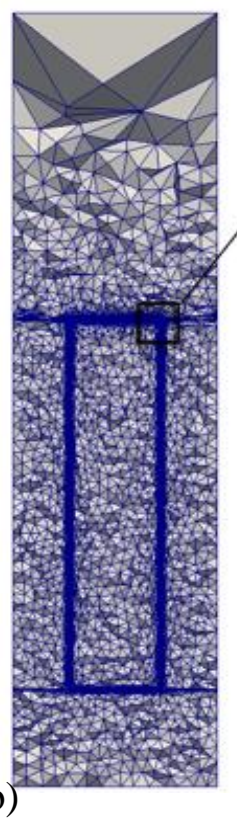

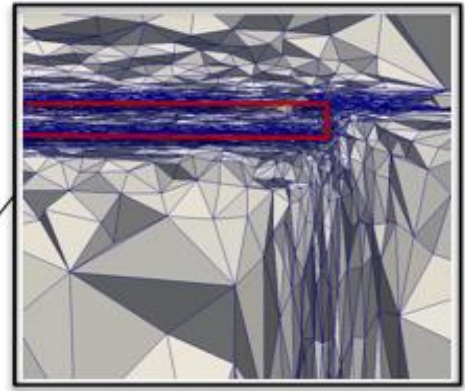

(c)

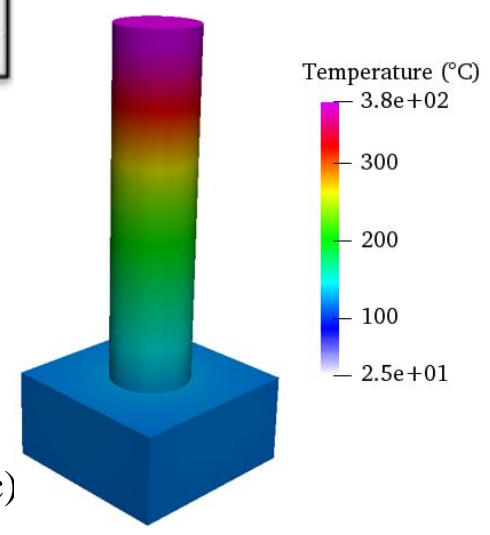

Fig. 6. Thermal simulation of the cylinder construction using the multi-layer "multi-shot" approach $\left(n^{\mathrm{l}}=5, n^{\mathrm{h}}=5\right)$. (a) Calculated temperature distribution at the end of the process in the whole domain, with a view of the finite element mesh in non-exposed powder and gas; (b) mesh distribution in the longitudinal median section of the domain (yz section plane by $x=0$ ) zoom at the top of the part with red contour line around the super-layer zone; (c) temperature distribution in the part only 
1 The computation times of the different methods for the entire construction are indicated in Table

23 , all of them being obtained by parallel calculations performed on 28 cores. They show

3 significant reduction when $n^{1}$ is 15 for the two super-layer strategies. The time gain is near 3

4 for $n^{h}=15$ ("multi-shot" deposition) compared with the reference case, and more than 6 for

$5 n^{h}=1$ ("one-shot" deposition), but with a significant decrease in accuracy. In total,

6 considering computation time and prediction quality, the super-layer strategy shows excellent

7 performance with the "multi-shot" method, even when using high values for $n^{1}$, such as 15 .

8 Table 3

9 Computation time for different sub-layers. 28 core parallel computations

\begin{tabular}{ccccc}
\hline$n^{\mathrm{l}}$ & 5 & 5 & 15 & 15 \\
\hline$n^{\mathrm{h}}$ & 5 & 1 & 15 & 1 \\
\hline Number of super-layers & 192 & 192 & 64 & 64 \\
\hline$T$ at point $B^{\prime}$ & 384 & 324 & 375 & 270 \\
\hline Number of Nodes & 0.46 & 0.46 & 0.52 & 0.52
\end{tabular}

\begin{tabular}{cccccc} 
Final mesh & $\left(\times 10^{6}\right)$ & & & & \\
\cline { 2 - 5 } information & $\begin{array}{c}\text { Number of } \\
\text { Elements } \\
\left(\times 10^{6}\right)\end{array}$ & 2.68 & 2.70 & 2.98 & 2.99 \\
& & & & \\
\hline
\end{tabular}

\begin{tabular}{ccccc}
\hline Computation time $(\min )$ & 10202 & 4563 & 3541 & 1595 \\
\hline Time gain & - & 2.24 & 2.88 & 6.39 \\
\hline
\end{tabular}

\section{COMPLEX APPLICATION}

12 The L-PBF manufacturing of an impellor, made of Ti-6Al-4V alloy is now considered. A similar geometry was studied in the past and reported in a previous paper [12]. However, the part studied here is twice as big as the previous one, which is more appropriate to evaluate the efficiency and precision of the proposed super-layer strategy. The part geometry is given in Fig. 7(a). The thickness of the blades is $2 \mathrm{~mm}$ and a go-through hole (radius $R_{1}=2 \mathrm{~mm}$ ) locates at

17 the center of the geometry. The L-PBF construction consists of 960 layers for a total part height of $24 \mathrm{~mm}\left(\Delta z_{l}=25 \mu \mathrm{m}\right)$. The construction is performed on a substrate of dimension $60 \times 60 \mathrm{x}$ $2 \mathrm{~mm}^{3}$. Fig. 7(b) illustrates the global construction environment, including the non-exposed powder and the gas zone, as it is at the end of the simulation. 
(a)
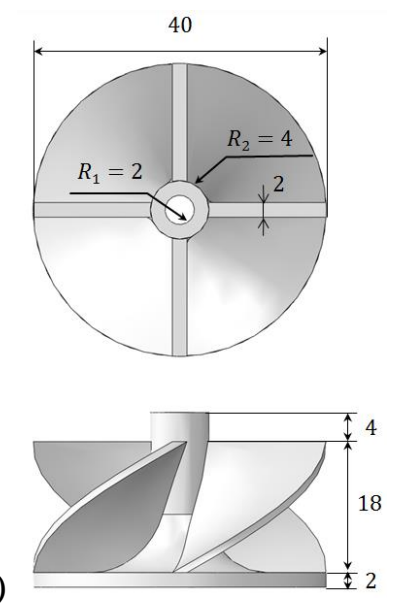

(b)

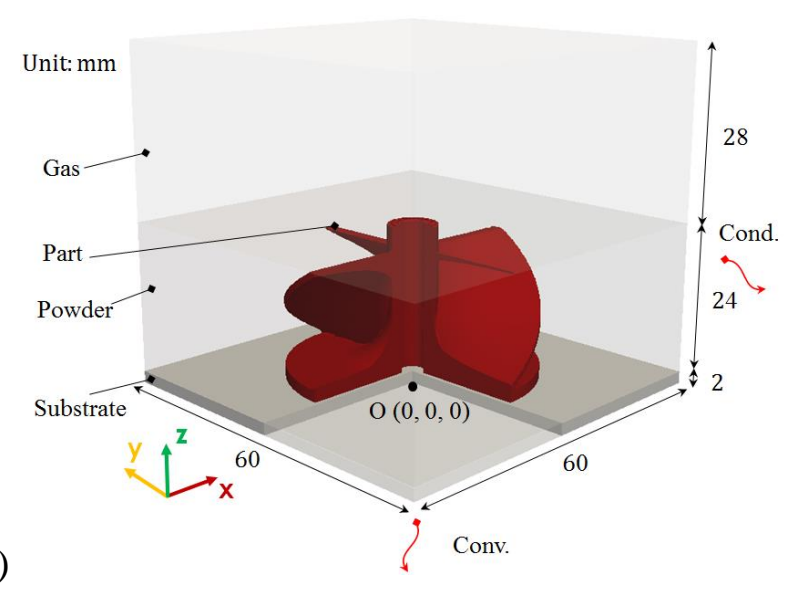

Fig. 7. Schematics of a complex L-PBF construction simulated at part scale showing (a) the part geometry; and (b) the global computation domain and its metallic and gas sub-domains as well as the boundary conditions (dimensions in $\mathrm{mm}$ )

1 The material properties are the same as in Section 5 (Table 1). The boundary conditions, the

2 initial conditions and the process parameters are given in Table 4. The reference frame is shown

3 in Fig. 7. Its origin is located at the center of the bottom face of the simulation domain, i.e. at

4 the basis of the substrate, hence coordinates within the domain evolving in the ranges

$5-30 \mathrm{~mm} \leq x \leq 30 \mathrm{~mm},-30 \mathrm{~mm} \leq y \leq 30 \mathrm{~mm}, 0 \mathrm{~mm} \leq z \leq 54 \mathrm{~mm}$.

6 Table 4 Heat exchanges and process parameters for the part shown in Fig. 7

\begin{tabular}{|c|c|c|}
\hline \multirow{5}{*}{$\begin{array}{l}\text { Heat } \\
\text { exchange }\end{array}$} & Temperature of machine environment, $T_{\text {ext }}$ & 25 \\
\hline & Initial temperature, $T_{0}\left[{ }^{\circ} \mathrm{C}\right]$ & 25 \\
\hline & Ambient temperature, $T_{\text {air }}\left[{ }^{\circ} \mathrm{C}\right]$ & 25 \\
\hline & Conductive coefficient, $h_{\text {cond }}\left[\mathrm{W} \mathrm{m}^{-2} \mathrm{~K}^{-1}\right]$ & 15 \\
\hline & Convection coefficient, $h_{\text {conv }}\left[\mathrm{Wm}^{-2} \mathrm{~K}^{-1}\right]$ & 15 \\
\hline \multirow{9}{*}{ Laser } & Nominal power, $P_{L}[\mathrm{~W}]$ & 200 \\
\hline & Scan speed, $v_{L}\left[\mathrm{~mm} \cdot \mathrm{s}^{-1}\right]$ & 1200 \\
\hline & Reflection coefficient, $R$ & 0.3 \\
\hline & Diameter of laser beam, $\phi_{L}[\mu \mathrm{m}]$ & 145 \\
\hline & hatch spacing, $H_{S}[\mu \mathrm{m}]$ & 100.05 \\
\hline & Thickness of powder layer, $\Delta z_{l}[\mu \mathrm{m}]$ & 25 \\
\hline & Deposited height, $\Delta z_{p, \max }[\mathrm{mm}]$ & 24 (960 layers) \\
\hline & Scaling factor, $\alpha$ & 500 \\
\hline & Inter-layer dwell time, $t_{d w e l l}^{l}[\mathrm{~s}]$ & 15 \\
\hline
\end{tabular}


16.1 Simulated temperature evolution: impact of $n^{1}$ in the super-layer method

2 In this section, the "multi-shot" super-layer method with different $n^{1}$ values is compared to the

3 "one-shot" method in terms of temperature evolution and distribution.

4 Four temperature sensors are defined to make this comparison as shown in Fig. 8. The first two

5 sensors are on the top surface of the substrate: sensor A is located in the bottom of the central 6 powder zone, coordinates $(0,0,2) \mathrm{mm}$; sensor B is located at coordinates $(0,-3,2) \mathrm{mm}$. Two 7 additional sensors A1 and B1 are selected. They are initially in the gas zone, respectively $3 \mathrm{~mm}$ 8 above $\mathrm{A}$ and $\mathrm{B}$. As the construction front passes by, A1 is found in the non-exposed powder, 9 while B1 is found in the part material. To verify temperature distribution, two line sensors are selected through the whole model: a vertical one ( $\mathrm{CC}^{\prime}$, going through substrate, powder and gas

11 regions, with $\mathrm{C}=\mathrm{O}$ ) and a horizontal one ( $\mathrm{DD}^{\prime}$ through powder, part, powder, part and powder) 12 at height $z=22 \mathrm{~mm}$.

13

13

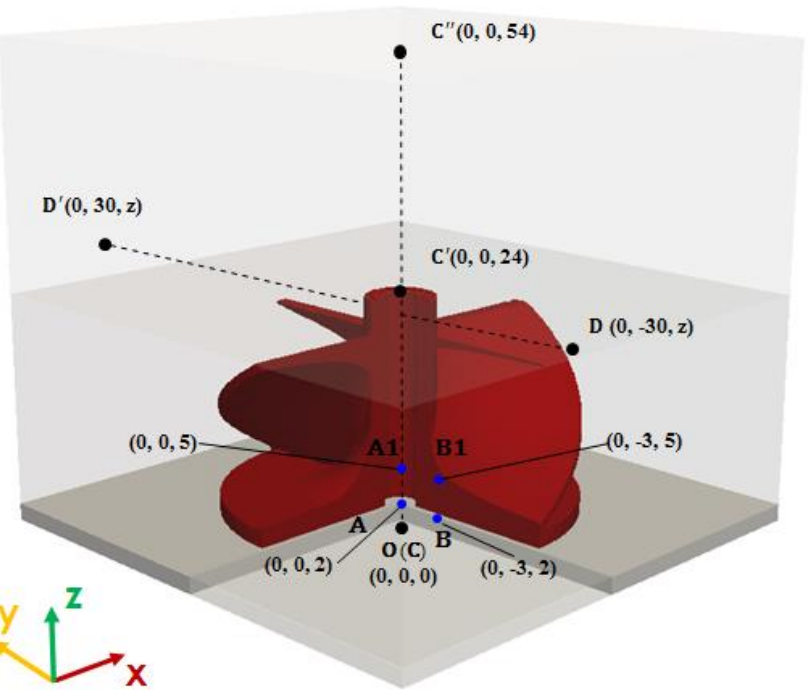

Fig. 8. Location of the selected points sensors, and line sensors along the vertical construction direction $\mathrm{CC}^{\prime \prime}\left(\mathrm{CC}^{\prime \prime}=\mathrm{OC}\right.$ ") and along the horizontal direction $\mathrm{DD}^{\prime}$ in a view representing the configuration after deposition of 960 layers

\subsubsection{Selection of the reference model for testing "multi-shot" method}

As the layer-by-layer strategy is too costly to simulate thousands of layers additions, the "multishot" method will be chosen to serve as a reference model for later multi-layer simulations. The objective of the present section is to validate this choice. To save computational cost and preserve precision, the deposition of the first 180 layers is studied for both the layer-by-layer 
1 and the "multi-shot" ( $\left.n^{\mathrm{l}}=5, n^{\mathrm{h}}=5\right)$ methods. The validation will be done through the 2 comparison of temperature evolution at sensors A and B (Fig. 8).

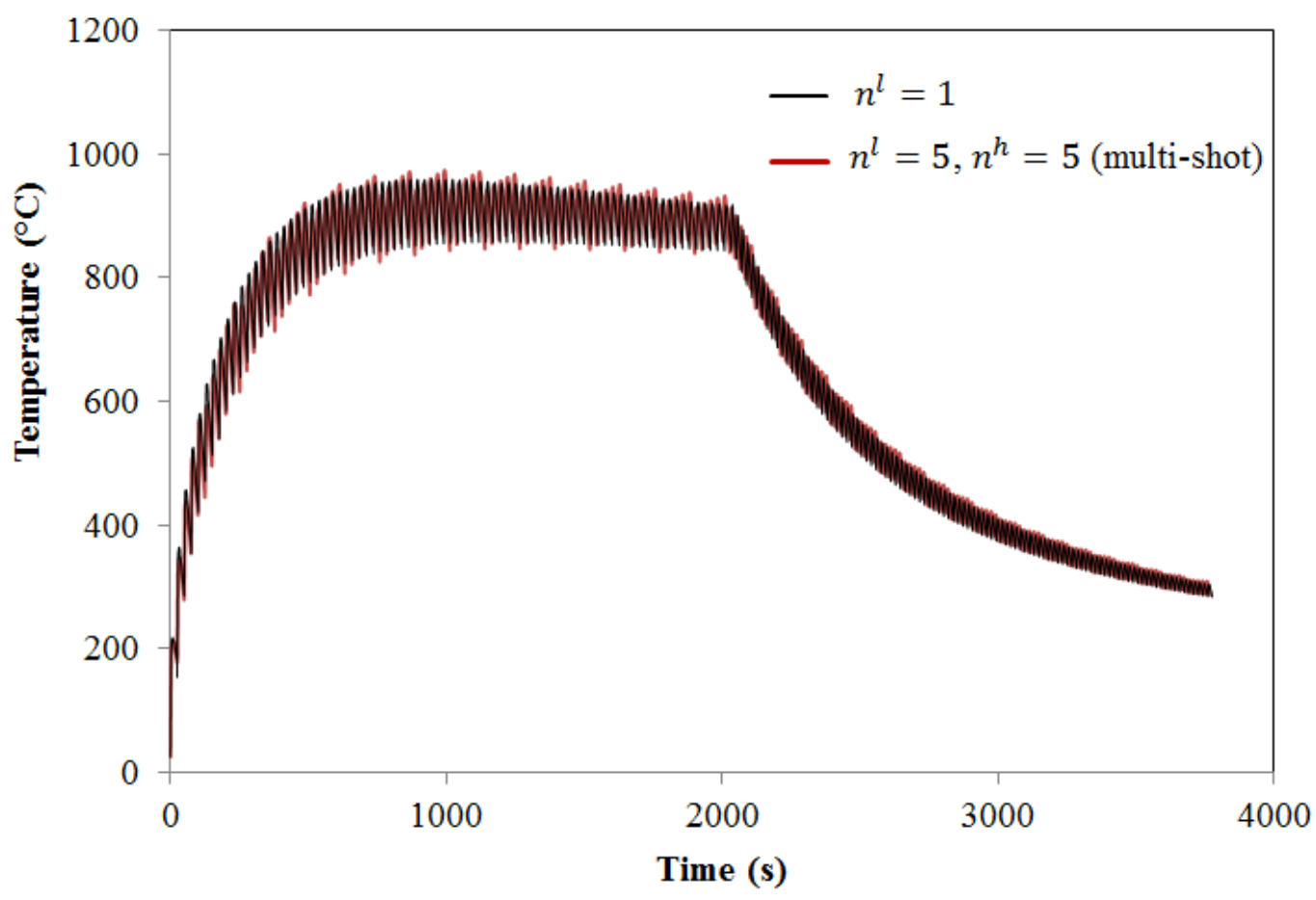

4

(a)

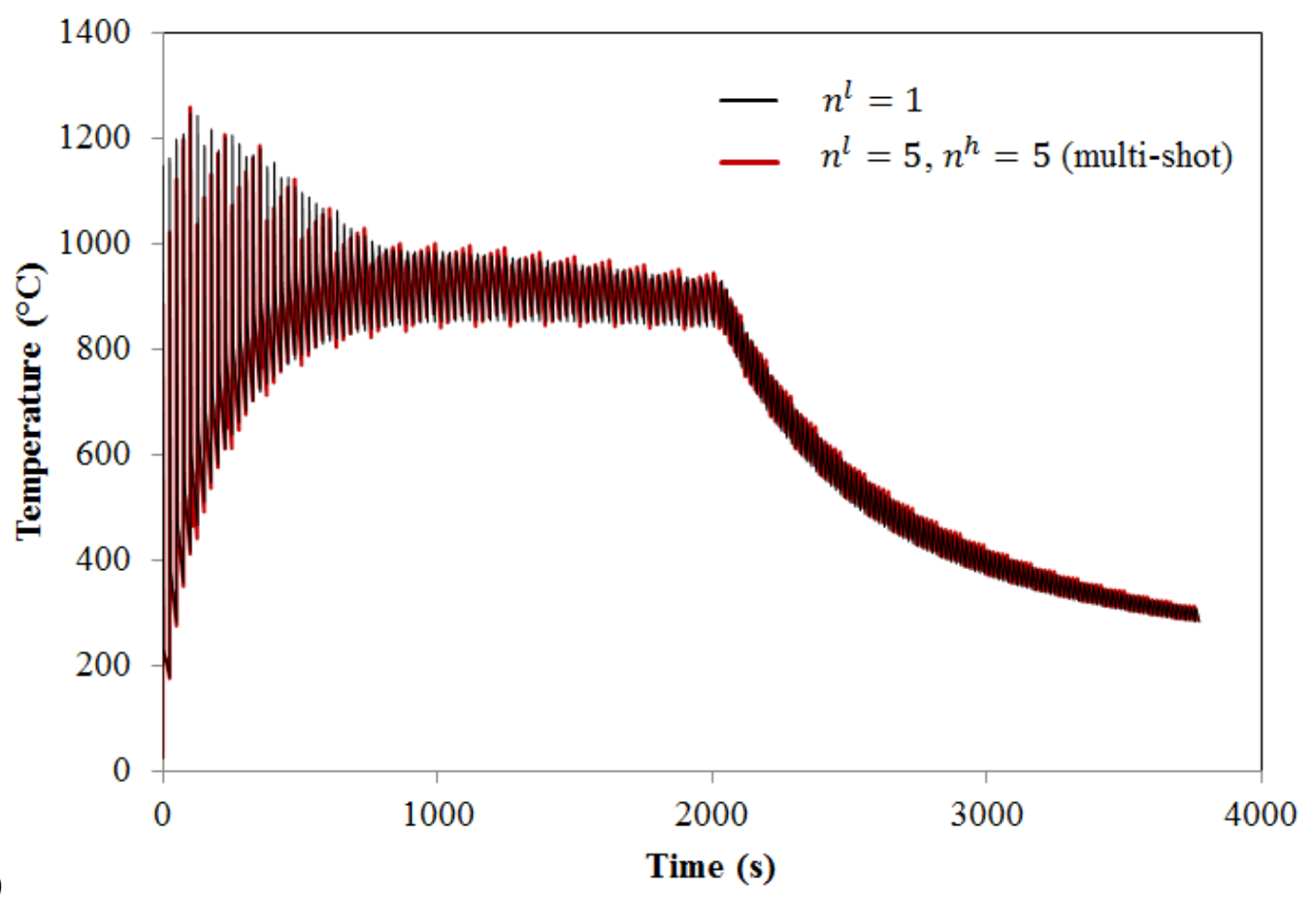

Fig. 9. Temperature evolutions: (a) sensor A (in powder zone); (b) sensor B (in part zone)

9 In Fig.9 (a), it can be seen that the "multi-shot" method with $\left(n^{\mathrm{l}}=5, n^{\mathrm{h}}=5\right)$ globally 10 provides a similar temperature evolution as for the layer-by-layer method. During the 
1 temperature increase, the "multi-shot" method reproduces very well the thermal cycling, with 2 almost the same cycling amplitude ( $\Delta T$ between successive max and min). The difference for this cycling amplitude is more perceptible during the plateau, where typically it is about $120{ }^{\circ} \mathrm{C}$ with $n^{l}=1$, and $150{ }^{\circ} \mathrm{C}$ with $n^{l}=5$. On the contrary, when the energy source goes far away from the sensor, the agreement regarding cycling is again excellent.

Similar trends are found for the second sensor B in the part zone, as shown in Fig. 9 (b). Because the heat conduction is much higher in the consolidated metal than in the powder bed, the temperature evolution at sensor B is very different from sensor $\mathrm{A}$ at the beginning of the construction. The layer-by-layer method shows a peak of temperature, expressing the heat cumulation during the deposition of the first layers, followed by a temperature decrease down to the same plateau at about $900{ }^{\circ} \mathrm{C}$ as for sensor A. This expresses that after the initial heat transient, the temperature becomes uniform in the consolidated part and in the powder bed in the vicinity of the two sensors. Regarding the initial transient regime, it can be seen in Fig. 9 (b) that the peak temperature characterizing the deposition of each "multi-shot" $\left(n^{\mathrm{l}}=5, n^{\mathrm{h}}=\right.$ 5 ) is in good agreement with the peaks obtained by the "layer by layer" method.

In total, following results already obtained in preceding sections, these results confirm the good accuracy of the "multi-shot" super-layer method. Hence, the "multi-shot" $\left(n^{\mathrm{l}}=5, n^{\mathrm{h}}=5\right)$ will be considered as the reference model for the entire assessment process.

\subsubsection{Comparison of the initial deposition at sensors A and B}

20 In this section, the study of the impact of $n^{l}$ is now extended to the entire process. Fig. 10 shows the calculated temperature evolution for both sensors A and B during the whole construction process. Because of a high computation cost, the single layer deposition $\left(n^{1}=1, n^{\mathrm{h}}=1\right)$ is not performed. As verified in subsection 6.1.1, the multi-shot deposition $\left(n^{1}=5, n^{\mathrm{h}}=5\right)$ is selected as the reference. A higher value of $n^{l}=15$ is adopted to verify two different super-layer strategies. The global evolution shows a first temperature increase stage, followed by a plateau and a slow cooling down for both the sensors in the powder and in the part, respectively. The slow temperature decrease ends at about 14000 s, after the deposition of 800 layers. At this time, the constructed height reaches $20 \mathrm{~mm}$ and the blades are fully printed. The last part of the construction begins. It consists in printing the top part of the axial shaft over a $4 \mathrm{~mm}$ height. This requires less laser heat input due to the quite small scanned surfaces, thus explaining the more efficient cooling rate at the position of the sensors. 
1 To compare the results, two zoomed sub-plots between the range of 0 and 2000s are given for 2 the "multi-shot" method of $n^{1}=5$ and $n^{1}=15$ in Fig. 10(a) and (b), respectively. In Fig. 10 3 (a) for the sensor $\mathrm{A}$ in the powder zone, the curve $\left(n^{\mathrm{l}}=15, n^{\mathrm{h}}=15\right)$ can cover the evolution 4 of $\left(n^{1}=5, n^{\mathrm{h}}=5\right)$, and the two curves overlap after 2000s during the cooling stage. Big 5 oscillations are found for the curve $\left(n^{1}=15, n^{\mathrm{h}}=1\right)$, while its average value is still around 6 the the evolution of $n^{1}=5$. Similar phenomena are found for the sensor in the part zone in Fig. 710 (b). Globally, the "multi-shot" method provides very similar average temperatures during 8 cycling, even with high $n^{1}=15$. The "one-shot" method shows big oscillation while maintaing 9 the convergence of the average temperature.

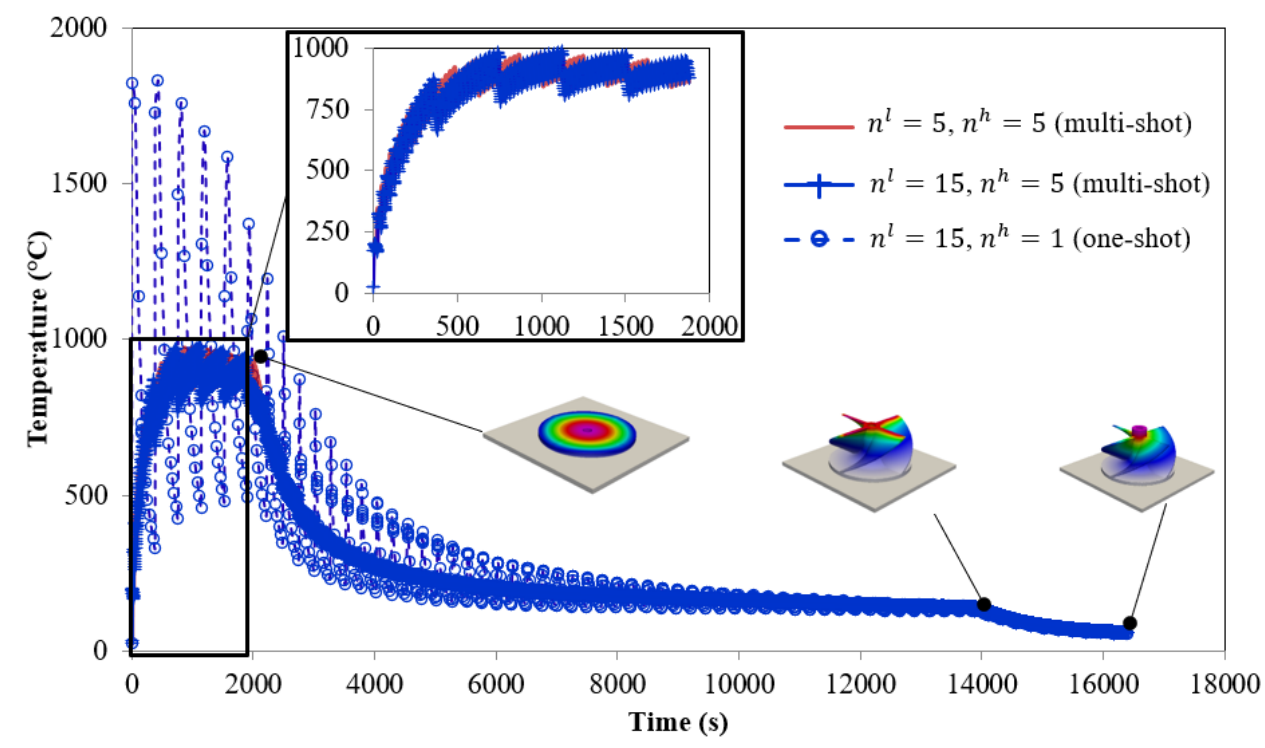

(a)

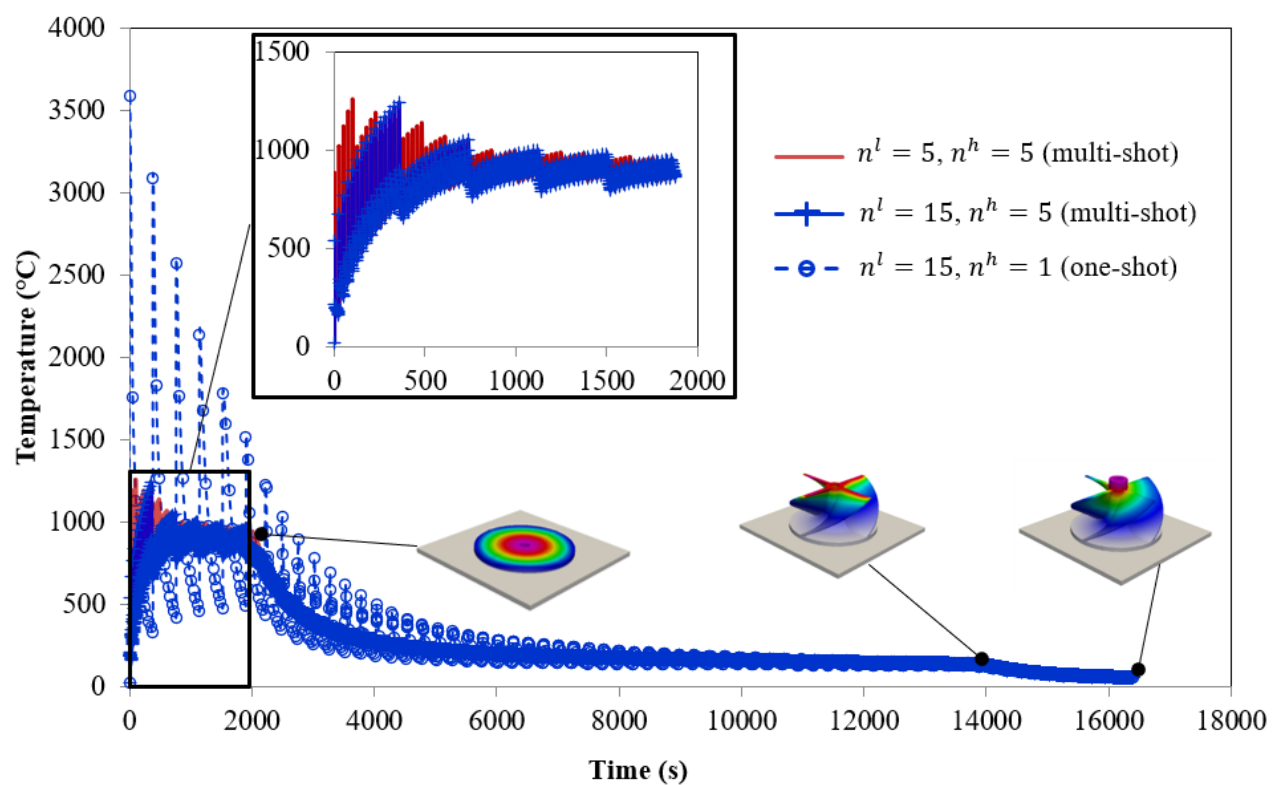

$11 \quad(b)$

Fig. 10. Temperature evolution of the entire process for (a) sensor A in the powder zone; and

(b) sensor B in the part zone, both positions defined in Fig. 8 
$1 \quad$ 6.1.3 Comparison during the construction process at sensors A1 and B1

2 To analyze further the response of the proposed model, two additional sensors $A 1(0,0,5)$ and $3 \mathrm{~B} 1(0,-3,5)$ are selected. They are initially located in the gas domain, $3 \mathrm{~mm}$ above sensors A 4 and B. Before the deposited material approaches the two sensors, big temporal oscillations are 5 found for the calculated temperature evolution, as shown in Figs 11(a) and 11(b) (the thin blue 6 curves). They express the heat cycling of the gas situated over the construction front, this cycling being associated with the multiple heat inputs during the deposition of the successive super-layers. It is reminded here that due to the level set method, a part of the laser energy supply affects the level set transition zone at the construction front, and thus is transferred to the gas. That explains the oscillations before the material deposition at the sensors A1 and B1.

11 However, it is important to note that despite the high temperature encountered by the argon gas 12 in the simulation, this represents a marginal energetic content as the ratio between the heat 13 capacity of the gas and the metal is very low: $\left(\rho^{\text {gas }} c_{p}^{\text {gas }}\right) /\left(\rho^{\text {metal }} c_{p}^{\text {metal }}\right)=5.2 \times 10^{-4}$. In orther words, as already studied in reference[12], the metal energetic input is almost not affected by this small loss tranferred to the gas. After the deposition of the $120^{\text {th }}$ layer, sensor A1 is embedded in the powder zone and sensor B1 is embedded in the workpiece. The temperature oscillations then rapidly disappear, and, like in previous analyses, a good convergence is observed between the calculations done with different $n^{l}$ values, for sensors in the powder and 19 in the part.

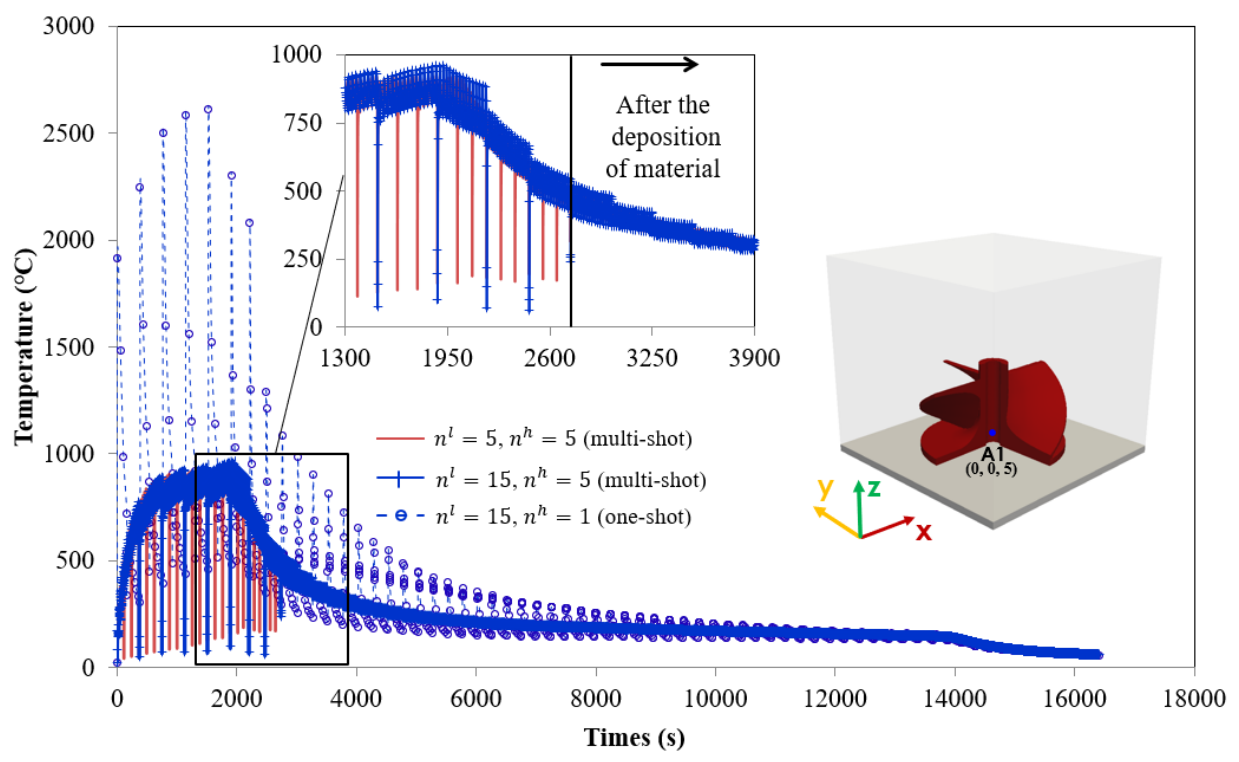




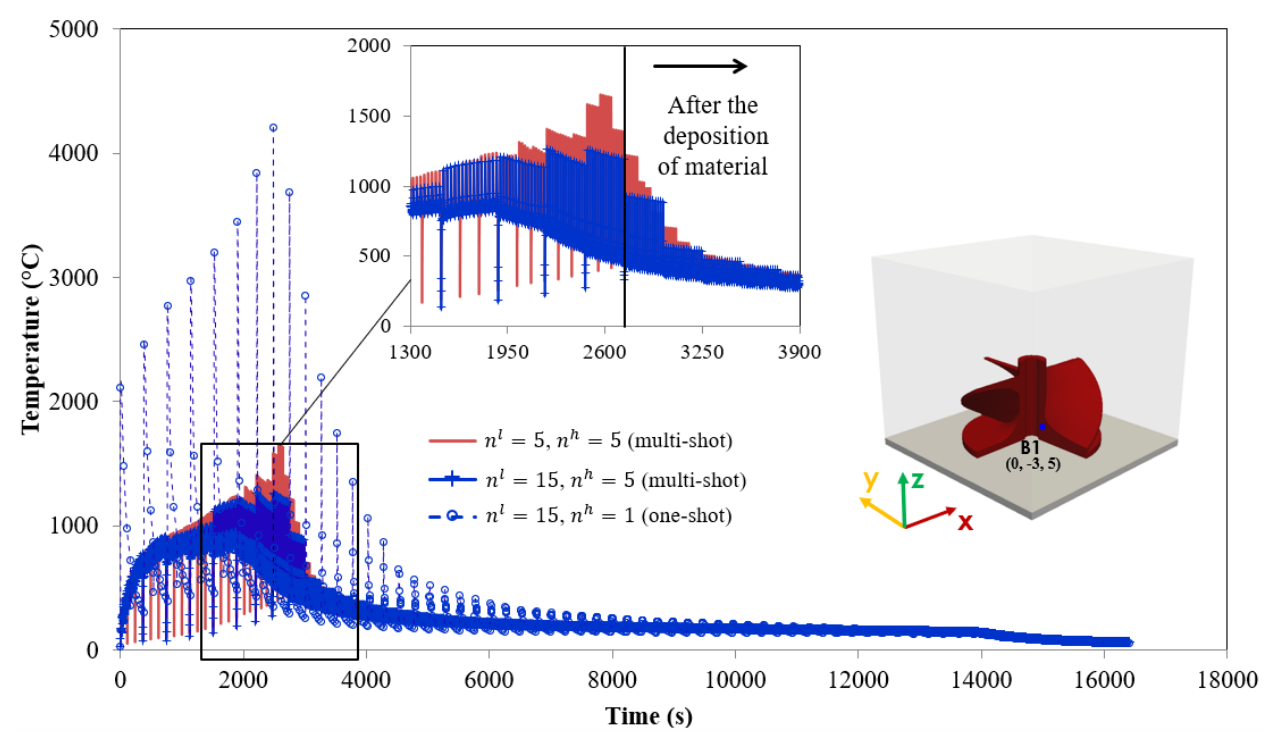

2 Fig. 11. Calculated temperature evolution during the entire process, using different $n^{l}$ values 3 (a) at sensor A1 in the gas first and in the powder later; and (b) at sensor B1 in the gas first and in the workpiece later. The black vertical line indicates the time at which the sensors A1 and $\mathrm{B} 1$ are included in the powder and the workpiece, respectively (coordinates in $\mathrm{mm}$ )

\subsection{Temperature distribution at the end of construction}

After the dwell time following the deposition of the last layer, the temperature distribution is obtained in the constructed part and in the whole model, as illustrated in Fig. 12(a), in which partial view of the adapted mesh is also shown. To verify the influence of $n^{l}$ on the temperature distribution, temperature profiles are plotted through the whole model as indicated in Fig. 8: a vertical one $\left(\mathrm{CC}^{\prime}\right.$, going through substrate, powder and gas regions, with $\left.\mathrm{C}=\mathrm{O}\right)$ and horizontal one (DD' through powder, part, powder, part and powder) at height $z=22 \mathrm{~mm}$. At the end of construction, the mesh distribution in the yz section plane is given. Like in Fig. 6. (b), the fine mesh is refined near the zero-isosurfaces of the two level sets $\psi$ and $\varphi$, as well as in the last deposited super-layer. Figs. 12(c) and 12(d) shows the different computed temperature profiles. It can be seen that results obtained with the "multi-shot" strategy, using $n^{l}=5$ and 15 , are very close, through the height of the part, and also along horizontal profiles.

19 Along the vertical direction, the temperature difference between the "multi-shot" method $\left(n^{1}=\right.$ $\left.15, n^{\mathrm{h}}=15\right)$ and the reference $\operatorname{case}\left(n^{\mathrm{l}}=5, n^{\mathrm{h}}=5\right)$ does not exceed $2^{\circ} \mathrm{C}$ (metal material 21 domain), which is excellent and confirms the good accuracy of the "multi-shot" strategy for conservation and distribution of energy. The fact that the prediction is not degraded with the augmentation of $n^{l}$ is all the more remarkable that, in this case and contrary to previous ones (the cylinder), the construction front is not perpendicular to the boundaries of the part: blades 
1 are significantly inclined with respect to the base plane. In contract, the temperature distribution 2 obtained with the "one-shot" strategy shows huge differences with the one of "multi-shot" 3 approach. This unsatisfying result of the "one-shot" strategy is in line with the results already

4 obtained for the plain straight cylinder (Fig. 5 (b)).

5

(a)

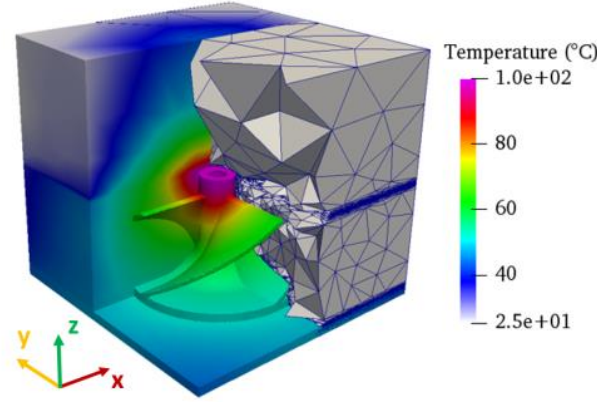

(b)
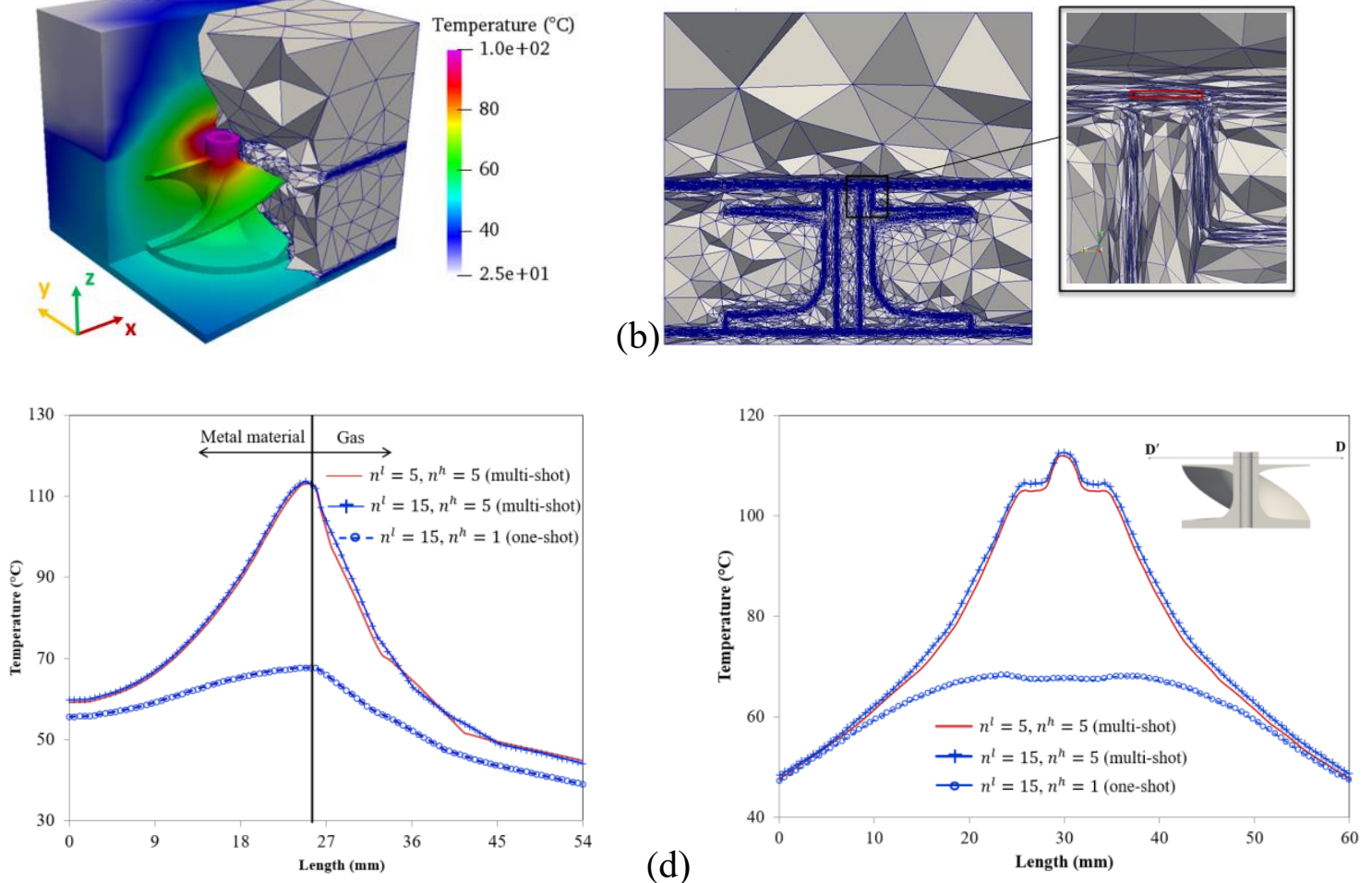

(d)

Fig. 12. Calculated temperature distribution at the end of the construction process with (a) 3D representation of the temperature field in the constructed part and surrounding powder, together with adapted mesh; (b) mesh distribution in the yz section plane of the domain, with zoom at the top of the part with red contour line along the super-layer zone; (c) and (d) obtained temperature profiles

As regards horizontal profiles, Fig. 12(d) reveals that the maximum temperature is always located in the central powder zone. Heat is actually confined in the axial zone, with a reduced axial and radial heat diffusion due to the low apparent conductivity of the powder zone. At the top of the part, close to the final construction front, this leads to a temperature excess in the powder of about $10{ }^{\circ} \mathrm{C}$ compared to the surrounding consolidated metal. It is also revealed that the different regions of the domain, when exposed to a similar radial heat flow, adopt extremely different temperature gradients. In particular, very low gradients looking like plateaus are observed in regions occupied by the consolidated metal. Here again, it can be noted that the "multi-shot" based super-layer method, associated with appropriate dynamic adaptive remeshing, complies well with regions having very different diffusivities. This is important as it is essential to consider the different domains (metal, powder) and their different properties when modelling heat transfer in L-PBF. Again, similar to the vertical line $\mathrm{CC}^{\prime}$, the temperature 
1 profile along the horizontal DD' obtained with the "one-shot" method shows a huge difference 2 with respect to the "multi-shot" method.

3

4 The computation time for the simulation of the entire model construction is given in Table 5

5 for 28 cores. Thanks to mesh adaptation, the element number is maintained around 2 millions.

6 The computation time reduces from 5175 minutes ( $~ 86 \mathrm{~h}$ ) when using $n^{l}=5$ to 1698 minutes$$
7
$$

\begin{tabular}{|c|c|c|c|c|}
\hline & $n^{l}$ & 5 & 15 & 15 \\
\hline & $n^{\mathrm{h}}$ & 5 & 15 & 1 \\
\hline \multicolumn{2}{|c|}{ Number of super-layers } & 192 & 64 & 64 \\
\hline \multicolumn{2}{|c|}{$T$ at point $C^{\prime}$} & 112 & 112 & 68 \\
\hline Final mesh & $\begin{array}{c}\text { Number of Nodes } \\
\left(\times 10^{6}\right)\end{array}$ & 0.41 & 0.41 & 0.42 \\
\hline information & $\begin{array}{l}\text { Number of Elements } \\
\qquad\left(\times 10^{6}\right)\end{array}$ & 2.36 & 2.36 & 2.40 \\
\hline \multicolumn{2}{|c|}{ Computation time (min) } & 5175 & 1698 & 301 \\
\hline \multicolumn{2}{|r|}{ Time gain } & - & 3.1 & 17.2 \\
\hline
\end{tabular}

In total, this application of the super-layer method to a complex L-PBF simulation case demonstrates that the proposed strategy, when properly designed using the "multi-shot" concept, is quite robust to predict the temperature distribution and its evolution during the process.

\section{CONCLUSIONS}

In this paper, an original solution scheme has been proposed to simulate the thermal aspects of PBF additive processes. It is based on the finite element method and combines energy based super-layer strategies, level set method, and mesh adaptation. In the present super-layer strategies, a series of $n^{l}$ material layers is deposited at the same time. Two different options have been tested with respect to heat flow. The "one-shot" option consists in heating each super- 
1 stages together. The "multi-shot" option includes as many heating steps as layers: $n^{h}=n^{l}$, generating thermal cycling at a higher frequency for each super-layer, and the already built metal underneath.

4 Compared to the reference layer-by-layer standard approach, it has been shown that the "multishot" super-layer method offers an excellent compromise, providing robust and accurate heat transfer predictions, while decreasing the computation time. The "one-shot" super-layer method offers the advantage of a faster time response, but at the cost of a degraded accuracy. In all cases, the super-layer methods offer a very efficient numerical tool to predict temperature evolution during the construction process. The combination with level sets and adaptive remeshing extends its capacity to obtain the temperature distribution not only in the part, but also in the non-exposed powder, which is essential for intricate geometries with possibly isolated powder zones. In final, using the super-layer method, simulating powder bed fusion process with thousands of layers and complex geometries is possible with a sustainable computation time and good precision.

\section{Acknowledgements}

The authors would like to thank Jesus Oswaldo Garcia for his internship works on the superlayer method at CEMEF (Sophia Antipolis, France) in the framework of a collaboration with the company AddUp (Clermont - Ferrand, France).

\section{REFERENCES}

[1] Murr, L.E., Gaytan, S.M., Ramirez, D.A., Martinez, E., Hernandez, J., Amato, K.N., Shindo, P.W., Medina, F.R. and Wicker R.B., 2012, "Metal Fabrication by Additive Manufacturing Using Laser and Electron Beam Melting Technologies," J. Mater. Sci. Technol., 28(1), pp.1-14. DOI: 10.1016/S1005-0302(12)60016-4

[2] Bandyopadhyay, A., and Heer, B., 2018, "Additive manufacturing of multi-material structures,” Mater. Sci. Eng. R Rep., 129, pp.1-16. DOI: 10.1016/j.mser.2018.04.001

[3] Körner, C., Attar, E., and Heinl, P., 2011, "Mesoscopic simulation of selective beam melting processes,” J. Mater. Process. Technol., 211, 978-987. DOI: 10.1016/j.jmatprotec.2010.12.016

[4] Khairallah, S.A., Anderson, A.T., Rubenchik, A., and King, W.E., 2016, "Laser powderbed fusion additive manufacturing: Physics of complex melt flow and formation mechanisms of pores, spatter, and denudation zones," Acta Mater., 108, pp. 36-45. DOI: 10.1016/j.actamat.2016.02.014 
[5] Bayat, M., Thanki, A., Mohanty, S., Witvrouw, A., Yang, S., Thorborg, J., Tiedje, N.S., Hattel, J.H., 2019, "Keyhole-induced porosities in Laser-based Powder Bed Fusion (L-PBF) of Ti6Al4V: High-fidelity modelling and experimental validation," Addit. Manuf., 30, p. 100835, DOI: 10.1016/j.addma.2019.100835

[6] Bayat M., Mohanty S., and Hattel, J. H., 2019, "Multiphysics modelling of lack-of-fusion voids formation and evolution in IN718 made by multi-track/multi-layer L-PBF," Int. J. Heat Mass Transf., 139, pp. 95-114. DOI: 10.1016/j.ijheatmasstransfer.2019.05.003

[7] Chen Q., Guillemot G., Gandin, C.-A., and Bellet, M., 2017, "Three-dimensional finite element thermomechanical modeling of additive manufacturing by selective laser melting for ceramic materials," Addit. Manuf., 16, pp. 124-137. DOI: 10.1016/j.addma.2017.02.005

[8] Chen Q., Guillemot G., Gandin, C.-A. and Bellet, M., 2018, "Numerical modelling of the impact of energy distribution and Marangoni surface tension on track shape in selective laser melting of ceramic material," Addit. Manuf., 21, pp. 713-723. DOI: 10.1016/j.addma.2018.03.003

[9] Saad, A., Gandin, Ch.-A., Bellet, M., 2015, "Temperature-based energy solver coupled with tabulated thermodynamic properties - Application to the prediction of macrosegregation in multicomponent alloys," Comput. Mater. Sci., 99, pp. 221-231. DOI: 10.1016/j.commatsci.2014.12.009

[10] Zhang, Y., Chen, Q., Guillemot, G., Gandin, Ch.-A. and Bellet, M., 2018, "Numerical modelling of fluid and solid thermomechanics in additive manufacturing by powder-bed fusion: Continuum and level set formulation applied to track- and part-scale simulations," Comptes Rendus - Mecanique, 346, pp. 1055-1071. DOI: /10.1016/j.crme.2018.08.008

[11] Moges, T., Ameta, G. and Witherell, P., 2019, "A Review of Model Inaccuracy and Parameter Uncertainty in Laser Powder Bed Fusion Models and Simulations," J. Manuf. Sci. Eng., 141(4), pp. 040801. DOI: 10.1115/1.4042789

[12] Zhang, Y., Guillemot, G., Bernacki, M. and Bellet, M., 2018, "Macroscopic thermal finite element modelling of additive metal manufacturing by selective laser melting process," Comput. Methods in Appl. Mech., 331, pp514-535. DOI: 10.1016/j.cma.2017.12.003

[13] Zhang, Y., Shapiro, V. and Witherell, P., 2020, "Scalable Thermal Simulation of Powder Bed Fusion." ASME 2020 International Design Engineering Technical Conferences and Computers and Information in Engineering Conference. ASME Digital Collection.

[14] Chiumenti, M. et al., 2017, "Numerical modelling and experimental validation in Selective Laser Melting,” Addit. Manuf., 18, pp. 171-185. DOI: 10.1016/j.addma.2017.09.002

[15] Zaeh, M.F., and Branner, G., 2010, "Investigations on residual stresses and deformations in selective laser melting," Prod. Eng. Res. Devel., 4, pp. 35-45. DOI: 10.1007/s11740-0090192-y

[16] Denlinger, E.R., Irwin, J. and Michaleris, P., 2014, "Thermomechanical modeling of additive manufacturing large parts," J. Manuf. Sci. Eng., 136, pp. 061007. DOI: $10.1115 / 1.4028669$ 
[17] Krola, T.A., Seidel, C., Schilp, J., Hofmann, M., Gan, W. and Zaeh, M.F., 2013, "Verification of structural simulation results of metal-based additive manufacturing by means of neutron diffraction," Phys. Procedia, 41, pp. 849-857. DOI: 10.1016/j.phpro.2013.03.158

[18] Peng, H., Ghasri-Khouzani, M., Gong, S., Attardo, R., Ostiguy, P., Gatrell, B. A., Budzinski, J., Tomonto, C., Neidig, J., Shankar, M.R., Billo, R., Go, D.B., Hoelzle, D., 2018, "Fast prediction of thermal distortion in metal powder bed fusion additive manufacturing: Part 1, a thermal circuit network model," Addit. Manuf., 22, 852-868. DOI: 10.1016/j.addma.2018.05.023

[19] Zhang, W., Tong, M., Harrison, N.M., 2019, "Resolution, energy and time dependency on layer scaling in finite element modelling of laser beam powder bed fusion additive manufacturing," Addit. Manuf., 28, pp. 610-620. DOI: doi.org/10.1016/j.addma.2019.05.002

[20] Bayat, M., Klingaa, C. G., Mohanty, S., De Baere, D., Thorborg, J., Tiedje, N. S., Hattel, J. H., 2020, "Part-scale thermo-mechanical modelling of distortions in Laser Powder Bed Fusion - Analysis of the sequential flash heating method with experimental validation," Addit. Manuf., 36, pp. 101508. DOI: /doi.org/10.1016/j.addma.2020.101508

[21] Li, C., Liu, Z.Y., Fang, X. Y., Guo, Y.B., 2018, "On the Simulation Scalability of Predicting Residual Stress and Distortion in Selective Laser Melting," J. Manuf. Sci. Eng., 140, pp.041013-1. DOI: doi.org/10.1115/1.4038893

[22] Shen N., and Chou K., 2012, "Thermal Modeling of Electron Beam Additive Manufacturing Process: Powder Sintering Effects". Proceedings of the ASME 2012 International Manufacturing Science and Engineering Conference. MSEC2012. June 4-8, 2012, Notre Dame, Indiana, USA.

[23] Sih, S.S., Barlow, J.W., 2004, "The prediction of the emissivity and thermal conductivity of powder beds," Particul Sci. Technol., 22(4), pp.427-440. DOI: $10.1080 / 02726350490501682 \mathrm{a}$

[24] Heigel, J.C., Michaleris, P., and Reutzel, E.W., 2015, "Thermo-mechanical model development and validation of directed energy deposition additive manufacturing of Ti-6Al4V,” Addit. Manuf., 5, pp.9-19. DOI: 10.1016/j.addma.2014.10.003

[25]Fachinotti, V.D., and Bellet M., 2006, "Linear tetrahedral finite elements for thermal shock problems," Internat. J. Numer. Methods Heat Fluid Flow, 16, pp.590-601. DOI: $10.1108 / 09615530610669120$ 


\section{Figure Captions List}

Fig. 1 Schematic of the level set approach for part-scale simulation

Fig. 2 Schematic time sequence to model a series of 5 layer additions, with indication of instants for material and energy supply for 3 methods: (a) layer-by-layer, with $n^{l}=1$ and $n^{h}=1$; (b) "multi-shot" super-layer, with $n^{l}=5$ and $n^{h}=5$; and (c) "one-shot" super-layer, with $n^{l}=5$ and $n^{h}=1\left(n^{l}\right.$ is the number of layers within each super-layer, $n^{h}$ is the number of heating steps for each super-layer)

Fig. 3 Geometry of the simulation system (dimensions in $\mathrm{mm}$ )

Fig. 4 Temperature evolution at location A during the deposition of the first 30 layers for the test case described by Fig. 3 and Table 1. Comparison is given between the layer-by-layer approach (thick black curve), the "one-shot" super-layer method (thin red line) and the "multi-shot" super-layer method (thick red line)

Fig. 5 Simulation of the construction of a vertical cylinder with the multi-shot super-layer strategy, using two different values for $n^{1}$ with (a) temperature evolution at sensor A and (b) temperature profile along the center line $\mathrm{BB}^{\prime}$ after the dwell time of the last layer

Fig. 6 Thermal simulation of the cylinder construction using the multi-layer "multi-shot" approach $\left(n^{\mathrm{l}}=5, n^{\mathrm{h}}=5\right)$. (a) Calculated temperature distribution at the end of the process in the whole domain, with a view of the finite element mesh in non-exposed powder and gas; (b) mesh distribution in the longitudinal median section of the domain (yz section plane by $x=0$ ) zoom at the top of the part with red contour line around the super-layer zone; (c) temperature distribution in the part only

Fig. 7 Schematics of a complex L-PBF construction simulated at part scale showing (a) the part geometry; and (b) the global computation domain and its metallic and gas sub-domains as well as the boundary conditions (dimensions in $\mathrm{mm}$ )

Fig. 8 Location of the selected points sensors, and line sensors along the vertical construction direction $\mathrm{CC}^{\prime \prime}\left(\mathrm{CC}^{\prime \prime}=\mathrm{OC}\right.$ ") and along the horizontal direction DD' in a view representing the configuration after deposition of 960 layers.

Fig. 9 Temperature evolutions: a) sensor A (in powder zone); b) sensor B (in part zone)

Fig. 10 Temperature evolution of the entire process for (a) sensor A in the powder zone; and (b) sensor B in the part zone, both positions defined in Fig. 8

Fig. 11 Calculated temperature evolution during the entire process, using different $n^{l}$ values (a) at sensor A1 in the gas first and in the powder later; and (b) at 
sensor B1 in the gas first and in the workpiece later. The black vertical line indicates the time at which the sensors A1 and B1 are included in the powder and the workpiece, respectively (coordinates in $\mathrm{mm}$ )

Fig. 12 Calculated temperature distribution at the end of the construction process with (a) 3D representation of the temperature field in the constructed part and surrounding powder, together with adapted mesh; (b) mesh distribution in the yz section plane of the domain, with zoom at the top of the part with red contour line along the super-layer zone; (c) and (d) obtained temperature profiles

\section{Table Caption List}

Table 1 Material properties, heat exchange and process parameters for the test case shown in Fig. 3 [7, 23, 24]

Table 2 Computation time for the simulation of a 30-layer deposition with 28 core parallel computation

Table 3 Computation time for different sub-layers. 28 core parallel computations

Table 4 Heat exchanges and process parameters for the part shown in Fig. 7

Table 5 Computation times for different $n^{l}$ numbers for the simulation of the impellor construction with 28 core parallel computation 